University of Wisconsin Milwaukee

UWM Digital Commons

Theses and Dissertations

May 2019

\title{
Derivation of Power System Module Metamodels for Early Shipboard Design Explorations
}

Rounak Siddaiah

University of Wisconsin-Milwaukee

Follow this and additional works at: https://dc.uwm.edu/etd

Part of the Electrical and Electronics Commons

\section{Recommended Citation}

Siddaiah, Rounak, "Derivation of Power System Module Metamodels for Early Shipboard Design Explorations" (2019). Theses and Dissertations. 2123.

https://dc.uwm.edu/etd/2123

This Thesis is brought to you for free and open access by UWM Digital Commons. It has been accepted for inclusion in Theses and Dissertations by an authorized administrator of UWM Digital Commons. For more information, please contact open-access@uwm.edu. 


\title{
DERIVATION OF POWER SYSTEM MODULE METAMODELS FOR EARLY SHIPBOARD DESIGN EXPLORATIONS
}

by

\author{
Rounak Siddaiah \\ A Thesis Submitted in \\ Partial Fulfillment of the \\ Requirements for the Degree of \\ Master of Science \\ in Engineering
}

at

The University of Wisconsin-Milwaukee

May 2019 


\title{
ABSTRACT \\ DERIVATION OF POWER SYSTEM MODULE METAMODELS FOR EARLY SHIPBOARD DESIGN EXPLORATIONS
}

\author{
by \\ Rounak Siddaiah \\ The University of Wisconsin-Milwaukee, 2019 \\ Under the Supervision of Professor Robert Cuzner
}

The U.S. Navy is currently challenged to develop new ship designs under compressed schedules. These ship designs must necessarily incorporate emerging technologies for high power energy conversion in order to enable smaller ship designs with a high degree of electrification and next generation electrified weapons. One way this challenge is being addressed is through development of collaborative concurrent design environment that allows for design space exploration across a wide range of implementation options. The most significant challenge is assurance of a dependable power and energy service via the shipboard Integrated Power and Energy System (IPES). The IPES is largely made up of interconnected power conversion and distribution equipment with allocated functionalities in order to meet demanding Quality of Power, Quality of Service and Survivability requirements. Feasible IPES implementations must fit within the ship hull constraints and must not violate limitations on ship displacement. This Thesis applies the theory of dependability to the use of scalable metamodels for power conversion and distribution equipment within a collaborative concurrent design environment to enable total ship set-based design outcomes that result implementable design specifications for procurement of equipment to be used in the final ship implementation.

More precisely, proposed MVDC-IPES implementations could be approached through multiobjective optimization techniques to minimize mass, volume, and power loss. The metaheuristic scheme is employed in this scenario to the active rectifier aspect of a power generation module 
(PGM), which interfaces between each generator and the MVDC bus and has a major impact on overall system space claim, displacement and heat. To begin, a specific, PGM-based, modular multilevel converter (MMC) topology and its Lowest Replaceable Units (LRUs) - the power electronic building blocks (PEBBs) along with the arm inductors - are addressed. The details of the virtual prototyping process applied to the LRUs are then compartmentalized. Finally, the thesis moves to outline a working implementation complete with input data and design results. The Module metamodel is designed to scale according to user defined cost objectives along pareto fronts representing the dimensions, weight, losses, reliability and cost of an corresponding optimal design for discrete points within a constrained design space.

These attributes will be selectable according to objectives set at the ship system level. In pursuant to the United States Navy's plans for an all-electric warship, highlights a framework for developing metaheuristic model-based scaling laws that can be applied to set-based designs of shipboard medium voltage direct current (MVDC) Integrated Power and Energy Systems (IPES). The best MVDC-IPES implementations will be based upon multi-objective optimized equipment that attempts to minimizes three objectives: mass, volume, and power loss. The active rectifier part of a power generation module (PGM), interfacing between each generator of the MVDC bus, will have a major impact on overall system space claim, displacement and heat. A specific modular multilevel converter (MMC) topological implementation of the PGM is addressed. A virtual prototyping process applied to the Lowest Replaceable Units (LRUs) within the PGM- the power electronic building blocks (PEBBs) along with the arm inductors - is described. In particular, selection of the overall design for the PEBB type was performed by evaluating discrete values of system DC bus voltages, inlet coolant water temperature, and generator frequency. 
(C) Copyright by Rounak Siddaiah, 2019

All Rights Reserved 


\section{DEDICATION}

Firstly, I would like to thank my professor Robert Cuzner for giving me opportunity to work with Metamodeling using Genetic Algorithm project. He guided me in every step of the entire research. He provided me with different exposures in the trending in the current industry I am thankful to my fellow colleague Thomas Nguyen from University of Wisconsin - Madison who helped me throughout this research. I would also like to thank Mahitha Voola for the moral support thought out my masters.

Last but not the least, I would like to thank my Mother, she is always the first person I call on for advice. She is not just my mother, but my counselor and friend and I will always grateful for all the sacrifice. 


\section{TABLE OF CONTENTS}

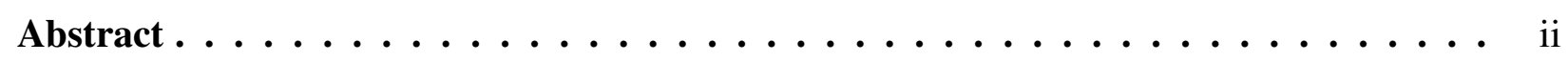

List of Figures $\ldots \ldots \ldots \ldots \ldots \ldots \ldots \ldots$

List of Tables ............................. ix

Glossary of Acronyms and Symbols . . . . . . . . . . . . . . .

Chapter 1: Introduction and Background . . . . . . . . . . . . . 1

Chapter 2: Shipboard Module Ontology . . . . . . . . . . . . . . . . 9

Chapter 3: Process for Development of Metamodel . . . . . . . . . . . . . . . 13

Chapter 4: Virtual Prototyping Process . . . . . . . . . . . . . . . 19

4.1 Optimization of Arm and Filter Inductors _ . . . . . . . . . . . 21

4.2 Design Metrics of the Inductor . . . . . . . . . . . . . . 23

4.2.1 The Magnetic Core: Storing Energy . . . . . . . . . . . . . . . 23

4.2.2 The Magnetic Core: Power Loss . . . . . . . . . . . . . . . . . 26

4.2.3 The Magnetic Core: Materials ... . . . . . . . . . . . 27

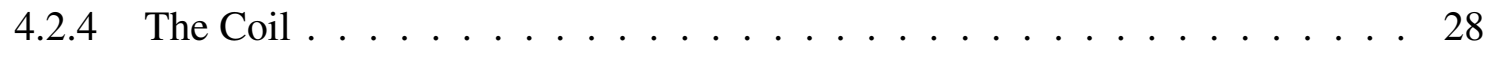

4.3 Selection of Design Process . . . . . . . . . . . . . . . . . 30 
4.3.1 Rationale behind Genetic Algorithm Selection . . . . . . . . . . . . . . . 30

4.4 Implementation of UI-Core Inductor Design Process . . . . . . . . . . . . . 32

$4.5 \quad P G M-R A$ Study and Analysis . . . . . . . . . . . . . 33

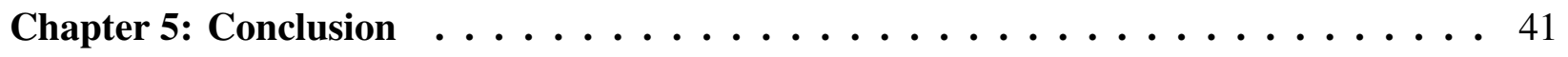

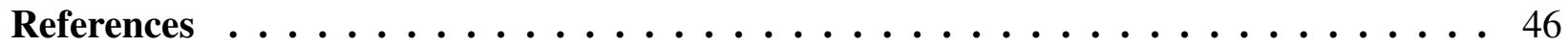




\section{LIST OF FIGURES}

1.1 Power Structure of a MMC . . . . . . . . . . . . . . . . . . . . . . 4

1.2 Sub-Optimal design of the Bay . . . . . . . . . . . . . . . . . 6

1.3 Optimal design of the Bay $\ldots \ldots \ldots \ldots \ldots$

2.1 Shipboard IPES architectures . . . . . . . . . . . . . . . . 9

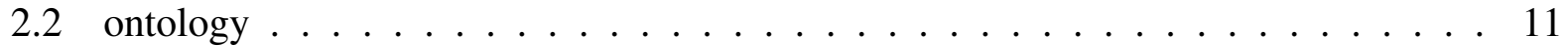

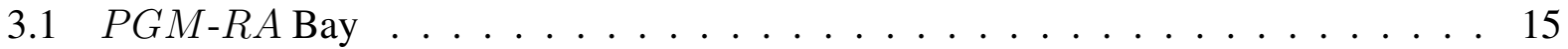

3.2 Overview of Metamodel generation process . . . . . . . . . . . . . 17

4.1 Virtual Prototyping Process(VPP-Step1) . . . . . . . . . . . . . . 20

4.2 Overall Virtual Prototyping Process including VPP-Step3 . . . . . . . . . . . . . 22

4.3 Organization of Genetic Algorithm Implementation . . . . . . . . . . . . . . 34

4.4 Organization of Fitness Function $\ldots \ldots \ldots$

4.5 Pareto Optimal Front . . . . . . . . . . . . . . . . 36

$4.6 P G M-R A$ Intrinsic Rated Power $\left(P_{i}\right)$ vs. $V_{D C}$, maximum $\rho$ designs $\ldots . . . . .38$

$4.7 \quad P G M-R A$ Power Density $(\rho)$ vs. $V_{D C}$, maximum $\rho$ designs $\ldots \ldots$. . . . . . 39

$4.8 P G M-R A$ Efficiency $(\eta)$ vs. $V_{D C}$ for, $\rho$ designs $\ldots \ldots \ldots$ 


\section{LIST OF TABLES}

4.1 Design Space and Constraints for Virtual Prototyping Process(VPP-Step2) . . . . 21

4.2 Design Variables for Inductor Optimization . . . . . . . . . . . . 32

4.3 Design Constraints for Inductor Optimization _ . . . . . . . . . . . 33 


\section{GLOSSARY OF ACRONYMS AND SYMBOLS}

IPS = Integrated Power System

IPES = Integrated Power and Energy System

$T M A=$ Thermal-Mechanical Assembly

$E M A=$ Electro-Mechanical Assembly

$E T A$ or $E T A_{i}=$ Electro-Thermal Assembly

$E A$ or $E A_{i}=$ Electrical/Electronic Assembly

$P C M=$ Power Conversion Module

$P G M=$ Power Generation Module

$P G M-R A=$ Power Generation Module Rectifier Assembly

PGM-SwA= Power Generation Module Switch Assembly

$P M M=$ Propulsion Motor Module

$P P M=$ Pulsed Power Module

$P D M=$ Power Distribution Module

$P E A$ or $P E A_{i}=$ Power Electronic Assembly

$L R U=$ Lowest Replaceable Unit

$P E B B$ or $P E B B_{i}=$ Power Electronic Building Block

$S S C B$ or $S S C B_{i}=$ Solid State Circuit Breaker

$N L S w$ or $N L S w_{i}=$ No Load Disconnect Switch

$E M C B$ or $E M C B_{i}=$ Electro-Mechanical Circuit Breaker

$A B T=$ Automatic Bus-Transfer Switch

$C A_{i}=$ Capacitor Assembly $I A_{i}=$ Inductor Assembly

$F A_{i}=$ Filter Assembly $\quad T A_{i}=$ Transformer Assembly

$\overrightarrow{\mathrm{M}_{\mathrm{u}}}=$ Module attribute $u \quad \overrightarrow{\mathbf{r}}_{s}=$ system constraints

$\overrightarrow{\mathbf{x}}_{s}=$ system design space $\quad \overrightarrow{\mathbf{k}}=$ design constants

$\overrightarrow{\mathbf{x}}_{d}=$ derived constraint $\quad O=$ objective function

$\overrightarrow{\mathrm{U}_{\mathrm{w}}}=$ LRU of type $w \quad \overrightarrow{\mathrm{D}_{\mathrm{w}}}=$ Drawer of type $w$ 
$\overrightarrow{\mathrm{C}_{\mathrm{w}}}=$ Compartment of type $w \quad \overrightarrow{\mathrm{B}_{\mathrm{w}}}=$ Bay of type $w$

$\overrightarrow{\mathbf{A}}=$ allocations $\quad \vec{\Psi}=$ features

$\vec{\alpha}=$ un-verified allocation $\quad \overrightarrow{\alpha^{*}}=$ verified allocation

$\overrightarrow{\alpha^{\prime}}=$ estimated allocation $\quad \overrightarrow{\hat{\alpha}}=$ behavioral allocation

$\overrightarrow{\mathbf{d}}_{\boldsymbol{v}_{x y z i}}=$ dielectric allocations $\left(\boldsymbol{v}=\overrightarrow{\mathrm{U}}_{w}, \overrightarrow{\mathrm{D}}_{w}, \overrightarrow{\mathrm{C}}_{w}, \overrightarrow{\mathrm{B}}_{w}\right)$

$\rightarrow \boldsymbol{v}_{x y z i}=$ thermal allocations $\left(\boldsymbol{v}=\overrightarrow{\mathrm{U}}_{w}, \overrightarrow{\mathrm{D}}_{w}, \overrightarrow{\mathrm{C}}_{w}, \overrightarrow{\mathrm{B}}_{w}\right)$

$\overrightarrow{\mathbf{f}}_{\boldsymbol{v}_{x y z i}}=$ structural allocations $\left(\boldsymbol{v}=\overrightarrow{\mathrm{U}}_{w}, \overrightarrow{\mathrm{D}}_{w}, \overrightarrow{\mathrm{C}}_{w}, \overrightarrow{\mathrm{B}}_{w}\right)$

$\overrightarrow{\mathbf{a}}_{\boldsymbol{v}_{x y z i}}=$ accessibility allocations $\left(\boldsymbol{v}=\overrightarrow{\mathrm{U}}_{w}, \overrightarrow{\mathrm{D}}_{w}, \overrightarrow{\mathrm{C}}_{w}, \overrightarrow{\mathrm{B}}_{w}\right)$

$\overrightarrow{\mathbf{c}}_{\boldsymbol{v}_{x y z i}}=$ interconnection allocations $\left(\boldsymbol{v}=\overrightarrow{\mathrm{U}}_{w}, \overrightarrow{\mathrm{D}}_{w}, \overrightarrow{\mathrm{C}}_{w}, \overrightarrow{\mathrm{B}}_{w}\right)$

PET $=$ Power Electronic Circuit Topology

$T M S=$ Thermal Management System

$P D T=$ Power Device Technolgy

$P E B B_{h}\left(T E P_{j}, T M S_{k}, P D T_{l}\right)=$ PEBB type $h$

belonging to $M_{T E P}$ type $j$ for TMS type $k$, PDT type $l$

$\vec{\Theta}=$ Genes associated with $\mathbf{U}_{w}-\mathbf{D}_{w}-\mathbf{C}_{w}-\mathbf{B}_{w}$ design space

$\overrightarrow{\mathbf{P}}_{\mathbf{B}_{v w}}=$ Type $v$ Module Bay of type $w$ Pareto optimal front

$\overrightarrow{\mathbf{x}}_{v}=$ design space $\mathbf{x}_{v} \subset \mathbf{x}_{s}$ applied to type $v$ Module

$\vec{r}_{v}=$ contraints $\boldsymbol{r}_{v} \subset \mathbf{r}_{s}$ for type $v$ Module

$N=$ total number of optimized designs for Module of type $v$

$n=$ optimized design $n$ for Module of type $v$

$M=$ total population of designs for Module of type $v$

$m=$ one design $m$ for Module of type $v$

$\rho=$ power density, $M W / m^{3} \quad \gamma=$ specific power, $k W / k g$

$\eta=$ efficiency $\quad \sigma=$ specific cost, $M W / U S D$

$\lambda^{-1}=$ reliability 


\section{CHAPTER 1}

\section{INTRODUCTION AND BACKGROUND}

Future highly electrified ship designs must necessarily incorporate emerging technologies for high power energy conversion in order to enable smaller ship designs with a high degree of electrification and high energy pulsed loads[1]. To address this challenge, a collaborative, concurrent design environment, called Smart Ship System Design, has been developed by the U.S. Navy in order to allow for a design space exploration across a wide range of MVDC power convertor implementations. Many of the questions related to design space explorations of MVAC to MVDC power convertor relate directly to the power conversion and distribution equipment implementations[2]. Scaling laws are needed that will predict size, weight, losses, cost and reliability as a function of design space variables associated with other design factors such as voltage, frequency and power levels, technology insertion, topological choices, etc. These scaling laws should produce reasonably accurate and realistic results and should represent a multi-disciplinary framework that is optimized to a range of objectives. This thesis proposes a methodology for producing metaheuristic model-based scaling laws [3] from a virtual prototyping approach that takes into account the discrete building blocks associated with multi-cell based power conversion and distribution equipment. The approach is demonstrated for a modular multi-level converter-based Medium Voltage AC to Medium Voltage DC active rectifier. The U.S. Navy is currently challenged to develop new ship designs under compressed schedules with simultaneous needs to increase electrification and reduce ship sizes [1], [4]. The load demand for such ships will necessarily exceed installed generational capability and therefore rely upon an Integrated Power and Energy System (IPES) as a network for power delivery between power/energy sources and loads that is re-configurable on demand [5], [6]. In order to ensure interoperability and survivability, many reconfiguration events will be autonomous[3]. The largest ship loads will be multi-MW ship propulsion and high energy weapon systems fed by a highly accessible and re-configurable connection to generational and 
short term energy sources through ship wide Medium Voltage dc (MVdc) buses. The effective implementations will require power electronics-based conversion and distribution of all power and energy between sources and loads. Present paradigms incorporate power conversion and distribution equipment within cabinets that include self-contained mitigation of surrounding environmentsuch as thermal, shock/vibration, voltage dielectric stand-off and electromagnetic compatibility. Future ship designs may embed this equipment into the ship hull construction. The demanding requirements rely upon emerging technologies, such a Wide Band Gap (WBG) power semiconductors, to enable MVdc interfacing power electronics with high power density [7].

Naval architects, ship builders and U.S. Navy procurement offices are undergoing an enormous paradigm shift when it comes the ship design and procurement process. Whereas the conventional focus for space/weight allocation has been focused on power generation and loads, where footprint of interconnecting switchgear cabinets are a relatively insignificant compared to the overall equipment space/weight budget, the massive introduction of power processing and re-configuring functionality significantly increases space/weight claim. While the ship designer recognizes that top-down, system level considerations must drive the design process, lack of attention to component level details (bottoms up considerations) can have disastrous effects. From the outset, the ship design must simultaneously address both top down and bottom up considerations. A completely new design approach is required, involving complex, dynamic interactions power/energy delivery networks combined with trade studies on space/weight claim, efficiency, reliability and cost. To address this concern, the U.S. Navy is developing a Smart Ship System Design(S3D) environment to enable concurrent, multi-disciplinary design [8], [9]. The S3D environment depends upon a catalog of equipment components, Leading Edge Architecture for Prototyping Systems (LEAPS) [10], to enable a Rapid Ship Design Environment (RSDE) that will apply a set-based design process to evaluation of thousands of potential ship designs [11], [12]. The LEAPS database includes program of record equipment with associated attributes such as dimensions, mass, losses, electrical, mechanical and thermal interfaces. Use of LEAPS within S3D enables simultaneous understanding of electrical, thermal, mechanical and layout aspects of the system design as it evolves. The 
S3D environment with LEAPS can be further exploited to implement autonomous design and scaling functions, such as cable sizing and routing and voltage/power rating scalability of equipment. For IPES-based design the vast majority of power and distribution Modules are developmental and introduction of power/energy density enabling technology, such as WBG, introducing risk into the ship design, procurement and integration. Metamodels for scalable power generation, conversion and distribution equipment have been introduced [13], [14], [15], [16]. These metamodels can be incorporated into the S3D catalog items to enable RSDE early concept studies. This thesis proposed an expansion of the metamodeling approach to include attributes that correlate to what would be needed for dependable ship design processes. The Navy is investigating in MVDC-IPES to enable ship electrical systems that will support electric propulsion and high energy weapons. [1]. The Navy has also invested heavily in the PEBB Concept for building up ship power conversion systems [2],[17],[18]. The Navy has developed Smart Ship System Design (S3D) concurrent engineering environment to support early concept explorations on ship system designs from electrical, mechanical, thermal and layout standpoints [19], [12]. The author of this thesis have proposed a meta-heuristic modeling approach that can be used to derive physics- based scaling laws [15]. Optimal implementation of PEBBs, along with determination of the optimal MVDC system bus voltage and the right topological approach to power conversion equipment, such as the PGM active rectifier, is a challenge. The MMC-based PGM active rectifier has particular value to "breaker-less" protection architectures and is well-suited to PEBB based implementations because of its modular nature [20], [21]. In prior work the author have investigated the derivation of MMC scaling laws derived from commercial equipment [14] and from an optimization process where maximum power density was the objective [22], [3]. In this thesis, the virtual prototyping process, from which scaling laws can be derived, incorporates a genetic algorithm to arrive at the best possible inductor designs, inlet coolant temperature and bus voltage (which establish the PGM rating)[23]. This thesis also evaluates viable and feasible PGM designs across a sytem MVDC voltage, inlet cooling water temperature, generator frequency and PEBB type design space. In terms of modular ship building design, the PEBB contribution is critical[24]. PEBBs are intended to be easily 


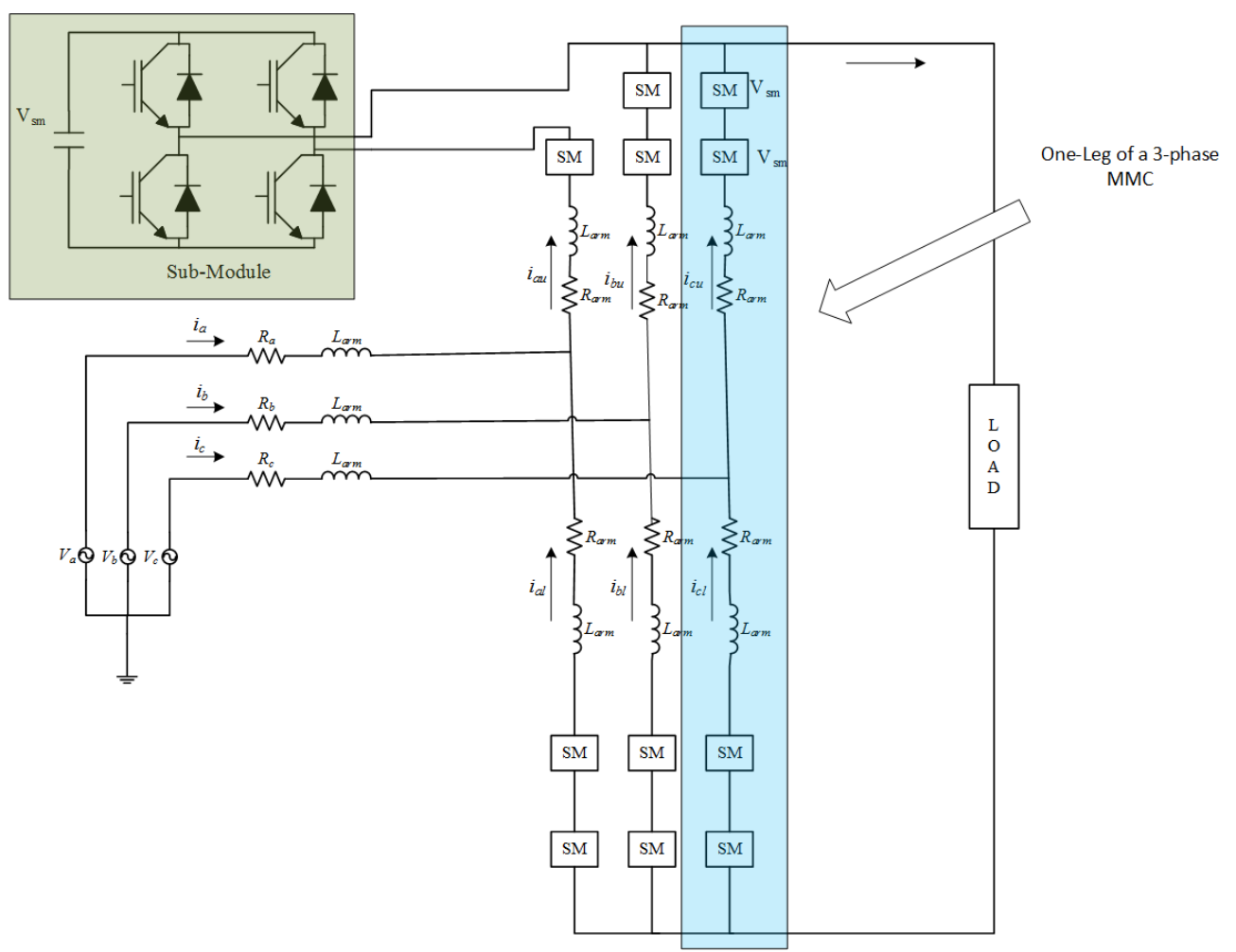

Figure 1.1: Power Structure of a MMC

replaced and to be configurable for multiple power converter types and functions throughout the ship. Furthermore, PEBB's can be designed with many different topologies and to accommodate different power semiconductor types and ratings. In this thesis MMC sub-modules are full-bridge converter-based PEBBs. Two PEBB types are considered, distinguished by their device ratings. They are based upon the 10kV, 240A [17] and 1.7kV, 400A Silicon Carbide (SiC) dual modules [18] presently being developed at Virginia Tech. The PEBB implementations follow these designs in layout but differ in two ways: (1.) They are fresh water liquid cooled, assuming that cold-plates are isolated from chassis and system ground through de-ionization of the coolant and electrical isolation of the coolant connects; (2.) The required sub-module capacitance for MMC operation (a result of the optimization process) is built into the PEBB. One-leg of the three phase MMC, shown in Fig. 1.1, is physically incorporated into a bay as shown in Fig. 1.2 and Fig. 1.3. The LRUs, arm inductors and PEBBs are arranged into drawers. Drawers are arranged into compartments and compartments are arranged into a single bay. The virtual prototyping process ensures the optimal 
arrangement of the LRUs, drawers and compartments into the bay. For example,1.2 shows a suboptimal arrangement (indicated by the amount of empty space). A more optimal arrangement is changed simply by rotating the arm inductor assemblies 90 degrees. The proposed solution to this problem, in brief, is to consider a holistic approach, evaluating all the input variables to the design of the MMC in the virtual prototyping process [3]. A multi-objective optimization approach using an evolutionary algorithm [25] can be applied at sub-level designs of the LRUs and PEBBs. More specifically, the genetic algorithm is implemented to search for a set of inductor dimensions, mass and power loss; qualifying designs enable a more focused selection for placing said components inside the cabinet ship bay. The desired result is a range of designs that are Pareto-optimized to Power Density $(\rho)$ in $M W / m^{3}$, Efficiency $(\eta)$ and Specific Power $(\gamma)$ in $k W / k g$. This leads into power and energy service via the shipboard Integrated Power and Energy System (IPES) also generating meta-models which integrates within Smart Ship Systems Design (S3D) the Rapid Ship Design Environment (RSDE) that will show thousands of potential set-based ship design options. The Navy has developed Smart Ship System Design (S3D) to support early concept explorations on ship system designs from electrical, mechanical, thermal and layout standpoints [8], [19], [26], [12]. S3D combines electrical, mechanical, thermal and thermodynamic design and simulation spaces with a three dimensional ship design and arrangement layout space. Work can proceed concurrently in these spaces. S3D includes connectivity to a database of components to enable the use of a common catalogue of equipment, each having interfaces and attributes for all of the design and simulation spaces [27], [28]. To support the Navy's ultimate goal of a Rapid Ship Design Environment (RSDE) [9] to enable early explorations of new ship designs incorporating new technologies, such as Wide Band Gap (WBG) power semiconductors into never-before built and qualified equipment, the equipment database should include scalable models for power conversion and distribution equipment [29], [7]. These models should help naval architects understand the value proposition of emerging technologies, such as WBG, to their ship designs. The models should also be capable explorations involving different topological implementations of the same power conversion or distribution function. For example Medium Voltage ac (MVac) to Medium 


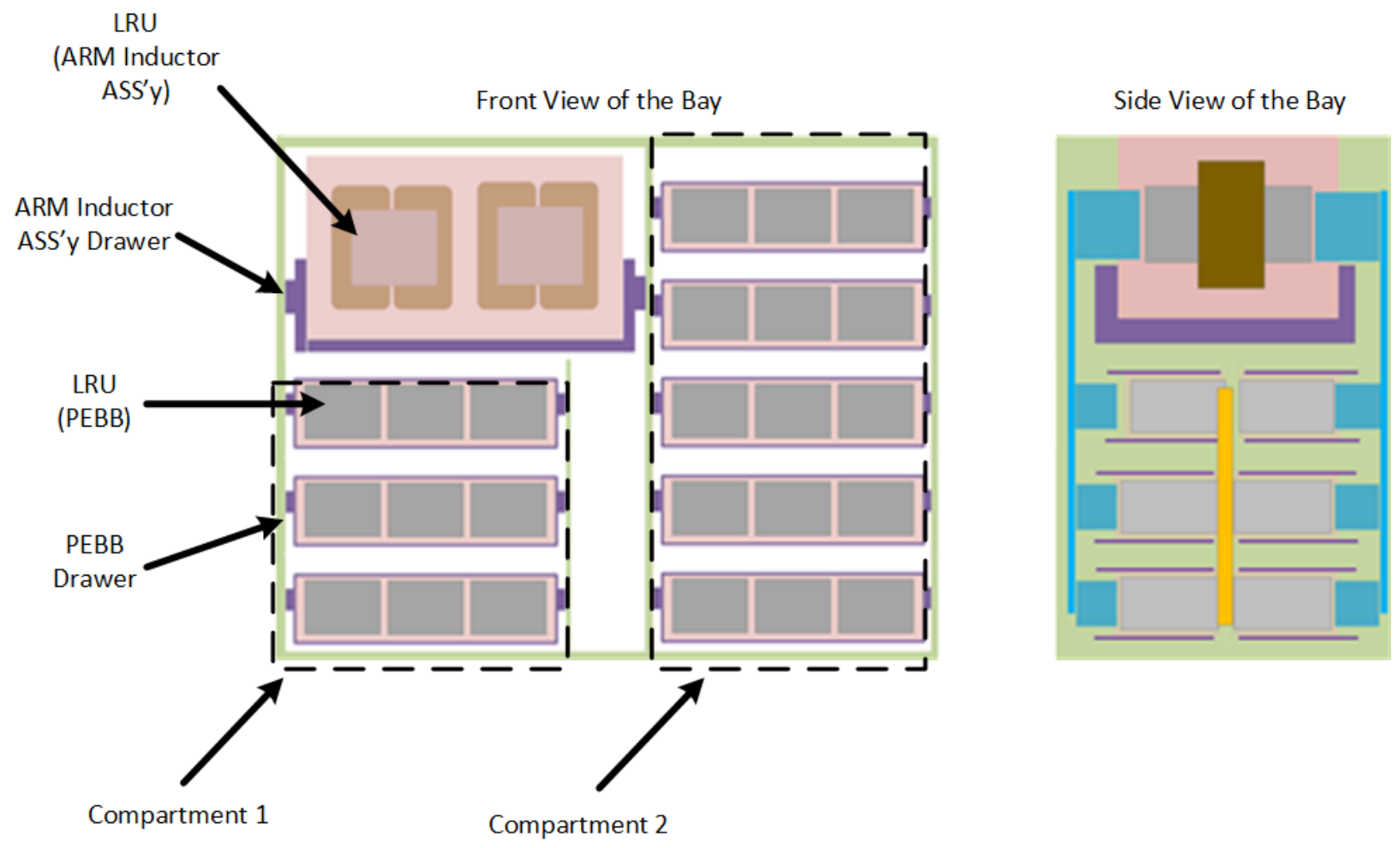

Figure 1.2: Sub-Optimal design of the Bay

Voltage dc (MVdc) power conversion can be implemented through various topologies, such as modular multi-level converter (MMC), cascaded H-Bridge, phase controlled-rectifiers and multilevel neutral point clamped (NPC) converters [30]. A useful modeling capability would enable inter-changeability of the topology within the same library component that will show how the equipment scales in size, weight, efficiency, etc. with topology choice. In its present state, S3D would only allow comparisons between static differences in equipment, such as size and weight, but there are plans to increase the functionality within the environment so that dynamic performance differences can also be explored [31].

The author has proposed a meta-heuristic modeling (metamodeling) approach that can be used to derive physics- based scaling laws for power conversion and distribution equipment [14], [15], [3]. Metamodels for scalable electromagnetic energy conversion devices (generators, motors) have already been introduced into the S3D environment [13], [16]. The development of metamodels for power conversion and distribution equipment faces a special challenge, due to the 


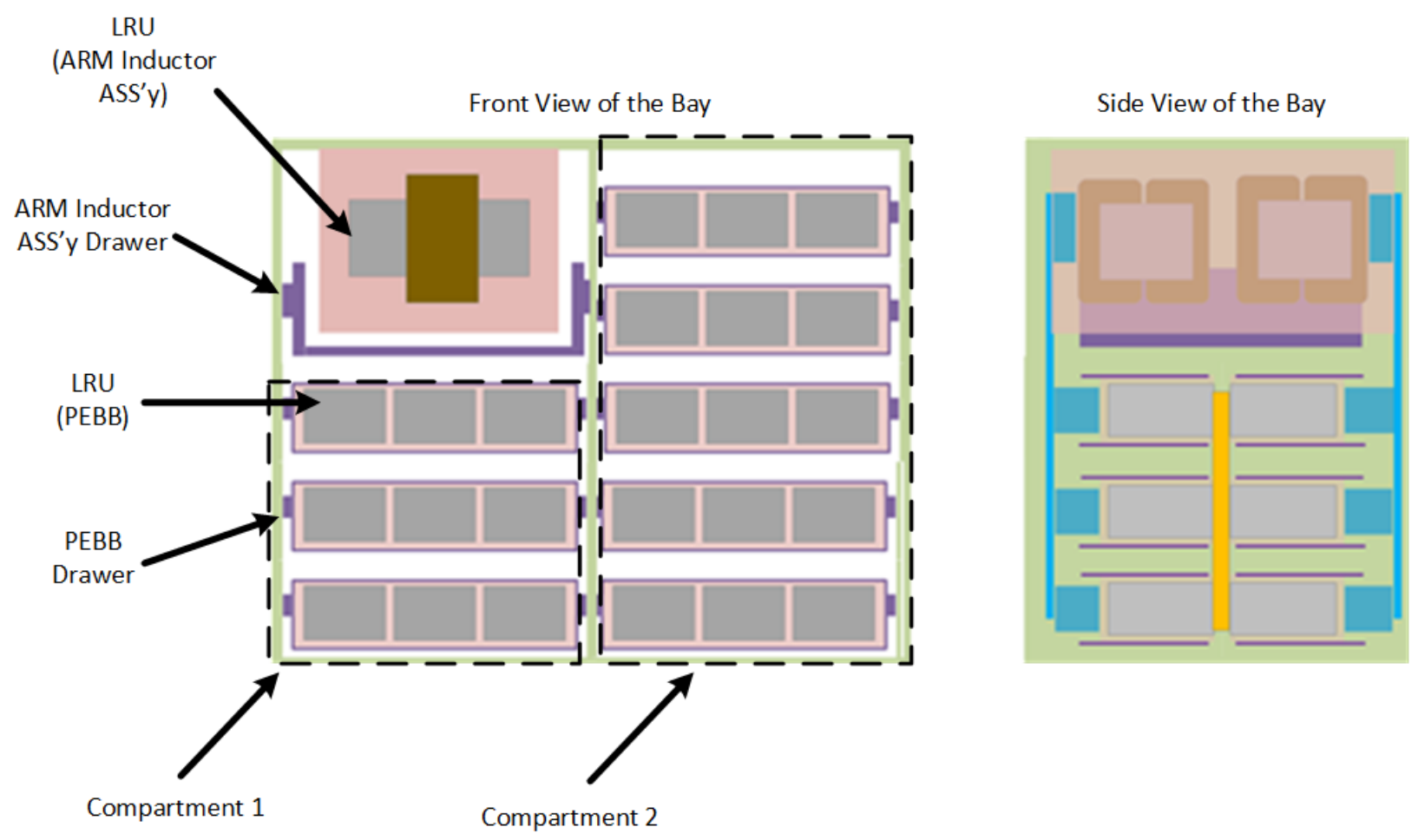

Figure 1.3: Optimal design of the Bay

multi-disciplinary nature of power electronics-based system designs, the strong influence of cabinet structure and accessibility on power density as the equipment scales up in size and the need to incorporate power electronic system implementations into enclosures that mitigate the impact of the power electronics on the surrounding thermal and electromagnetic compatibility (EMC) of the environment and vice-versa. The process for development of such equipment will involve a team of multi-disciplinary engineers, designing the constituent parts of the equipment against a set of customer and self-imposed requirements. Often, against the back-drop of demanding time schedules, cost constraints and risk reduction measures, an actual equipment implementation (if it exists) may not represent the optimum. Furthermore, the objectives against which the equipment is to be optimized may not be well defined and optimization objectves, such as power density and efficiency, are often in competition with each other. As a result, actual equipment implementations usually represent sub-optimal or non-optimized designs.

If scaling laws are derived from actual equipment, using behavioral data to extract trends or program of record equipment building blocks, then the conclusions derived from these scaling laws in 
an early ship concept exploration process may lead to overly conservative or incorrect conclusions regarding what can be achieved. These conclusions may result in disastrous results associated with the construction and life cycle costs once a ship design has been committed to. Furthermore, future shipboard electrification involves the concept of IPS or IPES, where the majority, if not all, of the power and energy is delivered through power electronic converters. In the case of MVdc distributions systems there are a range of possible topological implementations that are possible and, when coupled with a range of choices impacting the components making up the system, a host of possible feasible solutions. This thesis demonstrates the derivation of metamodel-based scaling laws for power conversion equipment. The metamodels are derived from a rigorous virtual prototyping process that ensures Pareto optimized solutions against multiple objectives. Metamodel development takes into account the practicalities associated with building equipment that can survive in a shipboard environment. The resultant metamodels retain a linkage to a pre-defined design space that enables determination the most feasible and viable electrical distribution architectures, topological implementations of individual power conversion and distribution functions, voltage and frequency levels, system level thermal management, etc. 


\section{CHAPTER 2}

\section{SHIPBOARD MODULE ONTOLOGY}

In conventional shipboard electrical distribution designs, the focus is on power generation and loads, with the footprint of interconnecting switchgear cabinets making up a relatively insignificant percentage of the overall equipment space/weight budget. However, with IPS/IPES-based electrical architectures, significant increase in space/weight claim by power conversion and distribution equipment accompanies the promised of dynamic re-configurability capabilities. The ship designer recognizes that top-down, system level considerations must drive the design process, but lack of attention to component level details (bottoms up considerations) can have disastrous effects. So the ship design must simultaneously address the system from the top down and from the bottom up. The S3D environment enables simultaneous understanding of electrical, thermal, mechanical and layout aspects of the system design as it evolves. The S3D environment can be further exploited to implement autonomous design and scaling functions, such as cable sizing and routing and voltage/power rating scalability of equipment. The end goal is to enable a RSDE that will apply a set-based design process to evaluation of thousands of potential ship designs [12], [32], [11].

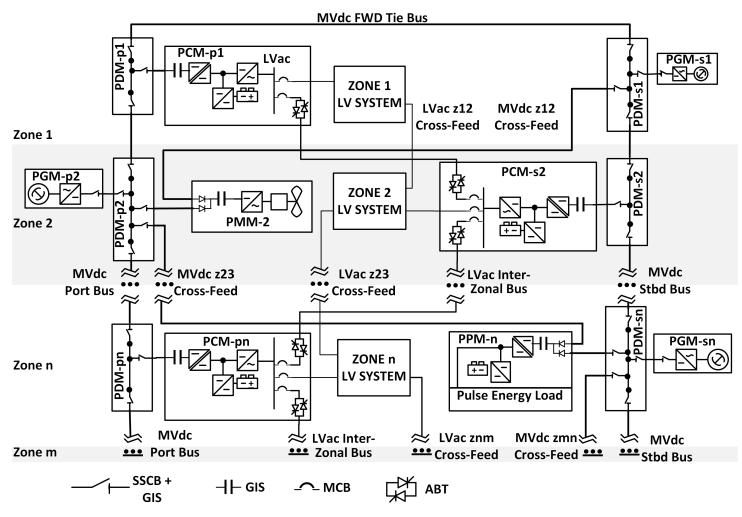

(a) Breaker-Based

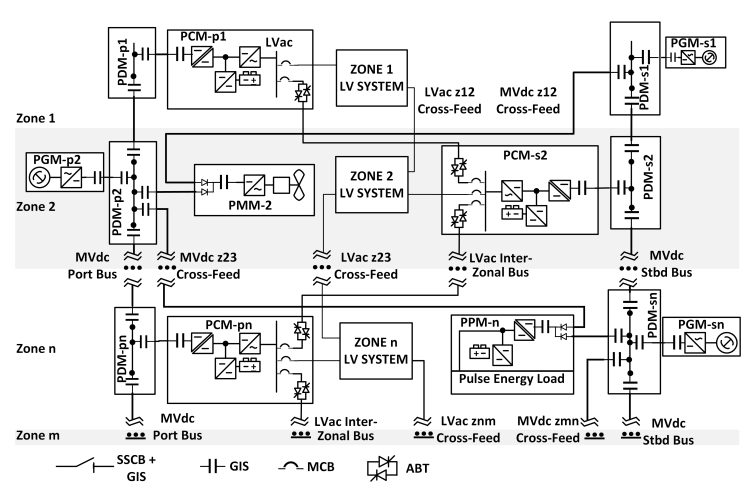

(b) Unit-Based

Figure 2.1: Shipboard IPES architectures 
Notional representations of two MVdc IPES architectures are shown in Fig. 2.1. All power and energy flows from installed generational sources and energy storage to loads through power electronics-based Modules. The MVdc interfacing Modules perform the following principal functions:

- The Power Generation Module (PGM) converts chemical energy to MVdc electrical energy

- The Power Conversion Module (PCM) converts MVdc electrical energy to inter-zonal LVdc distribution sytems.

- The Power Distribution Module $(P D M)$ longitudinally Distributes zonal MVdc power and energy

- The Propulsion Motor Module (PMM) converts MVdc electrical energy to mechanical propulsion energy

- The Pulsed Power Module (PPM) converts MVdc electrical energy to projectile or directed energy weaponry

A wide range of electrical architectures can be enabled through the optimal decomposition of required IPS/IPES functionalites to the Modules [30], [33]. For example, in the Breaker-Based protection architecture of Fig. 2.1a, the $P G M, P C M \mathrm{~s}, P M M \mathrm{~s}$ and $P P M$ s function principally as power and energy converters and the $P D M \mathrm{~s}$ are responsible for power and energy distribution and re-configuration and for detection, location, discrimination and isolation. On the other hand, the Unit-Based protection architecture of Fig. 2.1b expands the unit protection capabilities of the $P G M \mathrm{~s}$ and $P C M \mathrm{~s}$ for system level fault protection and coordinates $P G M \mathrm{~s}$ and $P C M \mathrm{~s}$ with the $P D M$ s for fault isolation and re-configuration. Both architectures can be implemented through a range of topological implementations of the $P G M \mathrm{~s}, P C M \mathrm{~s}$ and $P D M \mathrm{~s}$. Architectural and topological variations may also be driven by how Energy Storage Management $(E S M)$ is distributed among the Modules. For example, there are many options for distribution and location of 2.1 ESM will result in varying levels of power delivery reliability and survivability versus the contribution of the the IPS/IPES to ship sytem heat management, displacement, space claim and life cycle 


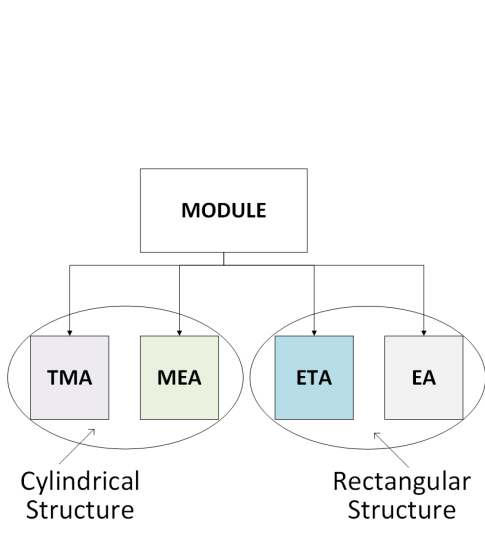

(a) Top level ontological structure

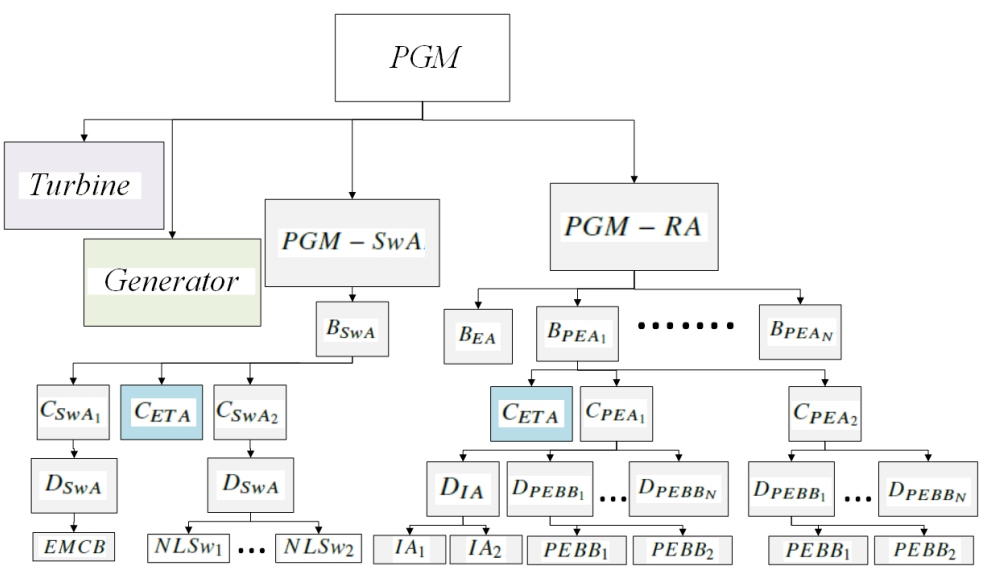

(b) Example of PGM ontology

Figure 2.2: ontology

cost. Module architectural and topological explorations should incorporate short term high energy demand, bi-directional energy flow and recovery during survivability events. Fig. 2.1 shows the allocation of ESM to the PCMs and PPMs but alternative architectures may distribute $E S M$ to the PDMs [34].

Since the Module implementation and functionality informs the shipboard architecture, the proposed approach enables the use of the Module for shipboard design explorations, which informs the top level Module ontology shown in Fig. 2.2. This ontology maintains linkages between the different design environments within S3D through four categories, as shown in Fig. 2.2(a) : Thermal-Mechanical Assembly ( $T M A)$, Electro-Mechanical Assembly ( $E M A)$, Electro-Thermal Assembly $(E T A)$ and Electrical Assembly $(E A)$. From an equipment-dimensioning perspective, the Module linkage to physical layout of the equipment sub-divides into two categories: cylindrical structures and rectangular structures. Scalable metamodels already exist within S3D to handle cylindrical structures (i.e. turbines, generators, motors). The rectangular structures will scale according to a Cartesian ( $x y z)$ coordinate system within an ontological structure that organizes the Lowest Replaceable Units $(L R U \mathrm{~s})\left(\overrightarrow{\mathrm{U}_{\mathrm{w}}}\right)$ into Drawers $\left(\overrightarrow{\mathrm{D}_{\mathrm{w}}}\right)$. Multiple Drawers having either the same or align-able aspect ratios organize into Compartments $\left(\overrightarrow{\mathrm{C}_{\mathrm{w}}}\right)$ and Compartments organize into Bays $\left(\overrightarrow{\mathrm{B}_{\mathrm{w}}}\right)$. The sub-script $w$ associated with $\overrightarrow{\mathrm{U}_{\mathrm{w}}}, \overrightarrow{\mathrm{D}_{\mathrm{w}}}, \overrightarrow{\mathrm{C}_{\mathrm{w}}}$ and $\overrightarrow{\mathrm{B}_{\mathrm{w}}}$ denotes the type, level and 
association of the $L R U$, Drawer, Compartment or Bay.

Fig. 2.2(b.) shows the ontology associated with a $P G M$. The $P G M$ Turbine and Generator are a TMA and $M E A$, respectively, and their scaling laws are not the subject of this thesis. However, the $P G M-S w A$ and $P G M-R A$ are EAs so they break out into hierarchies of Bays at the top level with Compartments containing Drawers, and Drawers containing LRUs. The PGM$S w A$ gives the $P G M$ the capability of being isolated from the Generator and MVdc system. As indicated in Fig. 2.1a, this this will be possible on the MVdc side through a series combination $S S C B(\mathrm{~s})$ and $N L S w(\mathrm{~s})$, if the $P G M-R A$ does not have the capability of driving its output current to zero when there is an MVdc short circuit fault (i.e. Breaker-Based protection architecture). If the $P G M-R A$ has the capability of driving output current to zero under when a short circuit occurs across the MVdc terminals (i.e. Unit-Based protection architecture) then fault isolation on the MVdc side is possible with only $N L S w(\mathrm{~s})$. Isolation on the Generator side of the circuit is possible through $E M C B(\mathrm{~s})$. The $P G M-R A$ for a Unit-Based protection architecture, represented in Fig. 2.2(b) houses the EMCB and NLSws in different Compartments $\left(C_{S w A_{1}}, C_{S w A_{2}}\right)$ to accommodate different dimensions and aspect ratios and to optimize arrangement within a single Bay $\left(B_{S w A}\right)$. 


\section{CHAPTER 3}

\section{PROCESS FOR DEVELOPMENT OF METAMODEL}

Prior work [14], [15] has focused on the development of a Virtual Prototyping Process (VPP) that enables optimal scaling within the constraints of a building-block based power converter approach utilizing the ontological structure of Fig. 2.2(b). In this work, the Module type and topology under study was the Full-Bridge MMC- or (FB-MMC)-based. The FB-MMC-based PGM-RA was selected because it juxtaposes a two-fold set of pros and cons representative of the entire approach that the U.S. Navy has adopted for future shipboard electrification. On the pro side, the FB-MMCbased $P G M-R A$ is (1.) a completely modular, building block-based system and (2.) a superior topology for the implementation of unit-based protection architectures. The cons associated with the FB-MMC-based $P G M-R A$ are (1.) the number active devices associated with the total number of PEBBs will be four times what is needed for any other non-isolated multi-level topology and (2.) The stacking of PEBBs to achieve higher voltage rated $P G M-R A$ s significantly erodes the power density that is represented by the power handling capability of an individual PEBB.

Regarding the first pro, the modularity of the MMC, not only are modular equipment implementations preferred in the shipboard environment, to enable maintainability, but the Navy has also invested heavily in the Power Electronic Building Block) PEBB Concept for building up ship power conversion systems [2],[17],[18]. The PEBB presents a convenient means for insertion of WBG power semiconductor technology into future shipboard systems. If a common PEBB is utilized across a wide range of Module types economies of scale can be achieved - increasing the viability of the architecture. The MMC is one of a small number of topologies for MV power conversion that really show-cases the benefit of PEBB. Regarding the second pro, if the MMC is made up of FB-based PEBBs then the circuit can inhibit internal DC link capacitor discharge during sudden fault application [35]. This significantly reduces the time required for Fault Isolation and Recovery (FIR) to within the time-trip response times of conventional EMCBs [36], 
[20],[37] compared to topologies such as the thyristor-based rectifier, which discharge capacitors into MVdc short circuit faults and require time on to order or $0.5-2$ seconds to re-energize the MVdc bus during FIR [38].

The PGM-RA PEA Bay structure of Fig. 3.1 corresponds to a $P G M-R A$ virtual prototype of one phase leg of an MMC. The Bay is the fundamental building block for Module scaling. For example, for the FB-MMC-based $P G M-R A$, the Bay represents all of the hardware associated with a functional phase leg optimized to a specific objective (i.e. minimum size, weight or cost or maximum efficiency or reliability). The assumed functional unit for the FB-MMC-based PGM$R A$ is three phase legs (three Bays) having an intrinsic rated power $\overrightarrow{\mathrm{P}}_{\mathrm{i}}$. If the $P G M$ Generator has six phases (two EMCB inputs) and one set of switch outputs (positive and negative rail) on the MVdc output then the $\overrightarrow{\mathrm{P}}_{\mathrm{i}}$ is associated with six Bays. $\overrightarrow{\mathrm{P}}_{\mathrm{i}}$ represents the highest achievable power for the FB-MMC-based ac-to-dc converter resulting from the highest achievable arm current subject to the Module type (v) design constraints $\left(\overrightarrow{\mathbf{r}}_{v}\right)$ and design space $\left(\overrightarrow{\mathbf{x}}_{v}\right)$. The VPP approach developed by the author is based upon the ontological structure of Fig. 2.2 with aim of developing a scalable $P G M-R A$ for the LEAPS database that is optimized to one of five objectives: power density $(\rho)$, specific power $(\gamma)$, efficiency $(\eta)$, reliability $\left(\lambda^{-1}\right)$ and specific cost $(\sigma)$ [39], [39].

As shown in Fig. 3.1, the LRUs are the required number PEBBs $\left(U_{P E B B}\right)$ to enable operation with a specified MVdc voltage rating $\left(V_{D C}\right)$, given the individual PEBB voltage capability and two arm inductor assemblies $\left(U_{I A}\right)$. Depending upon PEBB type constraints, either two or three PEBBs are arranged into a PEBB Drawer $\left(D_{P E B B}\right)$ and the two arm inductors are arranged into an $I A$ Drawer $\left(D_{I A}\right)$. As the PEBBs and inductors are arranged into Drawers and Drawers into compartments, the size and weight of the Bay is built up with allocations for dielectric standoff $\left(\overrightarrow{\mathbf{d}}_{x y z i}\right)$, thermal management $\left(\rightarrow \boldsymbol{v}_{x y z i}\right)$, frame and paneling structure $\left(\overrightarrow{\mathbf{f}}_{\boldsymbol{v}_{x y z i}}\right)$, accessibility $\left(\overrightarrow{\mathbf{a}} \boldsymbol{v}_{x y z i}\right)$, and cable and bus interconnections $\left(\overrightarrow{\mathbf{c}}_{\boldsymbol{v}_{x y z i}}\right)$ added to the space around PEBBs (in the $x y z$ planes) at the Drawer and Bay levels. The Drawer becomes a building block that is stacked up until a height constraint is reached, becoming a $P E A$ Compartment $\left(C_{P E A}\right)$. Any remaining drawers are stacked into adjacent $P E A$ Compartments until the phase leg is complete. The allocations 


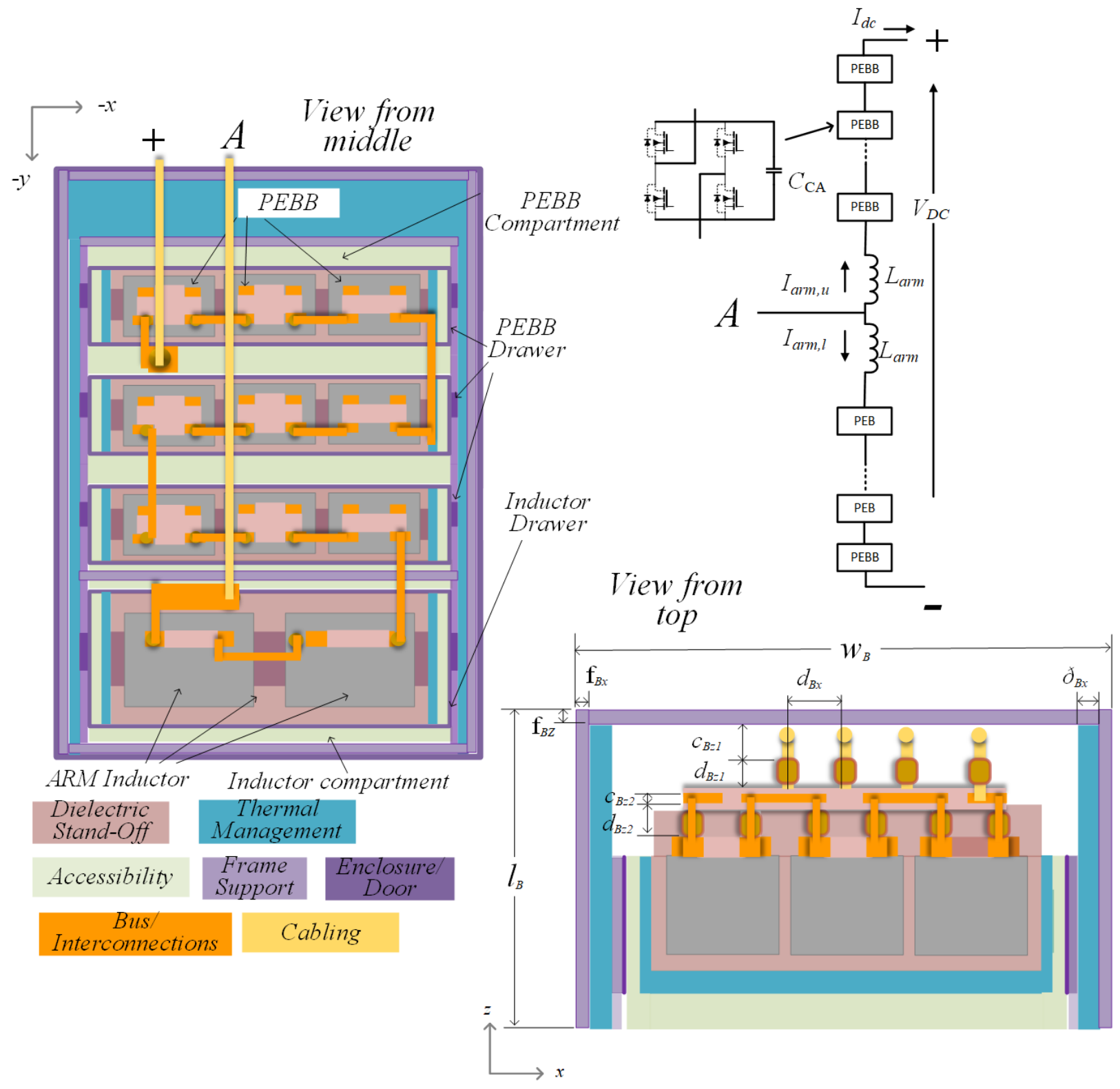

Figure 3.1: $P G M-R A$ Bay 


$$
\begin{aligned}
O=\min \quad & 1 / \alpha, 1 / \gamma^{-1}, 1 / \eta^{-1}, \lambda, 1 / \sigma \\
\text { over } \overrightarrow{\mathbf{x}}_{v}= & \vec{M}_{P E T} \in\left[P E T_{1}, P E T_{2}, \ldots, P E T_{f_{\max }}\right] \\
& \vec{M}_{T M S} \in\left[T M S_{1}, T M S_{2}, \ldots, T M S_{h_{\max }}\right] \\
& \vec{U}_{P E B B} \in\left[P E B B_{1}\left(T E P_{1}, T M S_{1}, P D T_{1}\right),\right. \\
& P E B B_{2}\left(T E P_{1}, T M S_{1}, P D T_{1}\right), \ldots, \\
& P E B B_{h_{\max }}\left(T E P_{j_{\max }}, T M S_{k_{\max }}, P D T_{\left.l_{\max }\right)}\right) \\
& \vec{T}_{A} \in[5,15,25,35] \\
& \vec{V}_{D C} \in[12,13,14, \ldots, 30] \\
& \vec{F}_{e} \in[60,120,180,240] \\
\text { for } \overrightarrow{\mathbf{r}}_{v}=\quad & h_{B_{w}} \leq \text { Maximum Height } \\
& l_{B_{w}} \leq \text { Maintainability Space } \\
& \Delta \mathrm{P} \leq \text { Module level } \Delta \mathrm{P}_{\max } \\
& \text { Flow Rate } \leq \text { Flow Rate constraint }
\end{aligned}
$$

account for the space and weight around Drawers and Compartments associated with a realistic Bay implementation having a modular, maintainable structure with appropriate mitigation for the electrical, thermal and mechanical shipboard environmental constraints. Allocations are added to around the Compartments at the Bay level resulting in its final outside dimensions and weight.

Fig. 3.2 shows the entire process for metamodel generation. The VPP receives as its inputs, $\overrightarrow{\mathbf{r}}_{v}$ and $\overrightarrow{\mathbf{x}}_{v}$ which are subsets of the shipboard design constraints $\overrightarrow{\mathbf{r}}_{s}$ and design space $\overrightarrow{\mathbf{x}}_{s}$. The VPP produces a $\rho-\gamma-\eta-\lambda^{-1}-\sigma$ Pareto optimized feature space of the Bay, $\overrightarrow{\mathbf{k}}\left[\overrightarrow{\mathbf{P}}_{\mathbf{B}_{w}}\left(\overrightarrow{\mathbf{x}}_{v}\right)\right]$, by executing the objective function of above equation.

The result is total of $5 \cdot N$ designs, where $N$ represents the total number maximum $\rho-\gamma-\eta-\lambda^{-1}-\sigma$ designs for every meaningful combination of design space elements listed in above equation.

The LRU and Drawer performance features $\vec{\Psi}\left[\overrightarrow{\mathbf{P}}_{\mathbf{U}_{w}}\left(\overrightarrow{\mathbf{x}}_{v}\right), \overrightarrow{\mathbf{P}}_{\mathbf{D}_{w}}\left(\overrightarrow{\mathbf{x}}_{v}\right)\right]$ and the parameters associated with the Pareto front of the performance space, $\overrightarrow{\mathbf{k}}\left[\overrightarrow{\mathbf{P}}_{\mathbf{U}_{w}}\left(\overrightarrow{\mathbf{x}}_{v}\right), \overrightarrow{\mathbf{P}}_{\mathbf{D}_{w}}\left(\overrightarrow{\mathbf{x}}_{v}\right)\right]$, are extracted out of $\overrightarrow{\mathbf{k}}\left[\overrightarrow{\mathbf{P}}_{\mathbf{B}_{w}}\left(\overrightarrow{\mathbf{x}}_{v}\right)\right]$ and mapped back to the VPP design space inputs $\mathbf{x}_{v}$. Examples of 


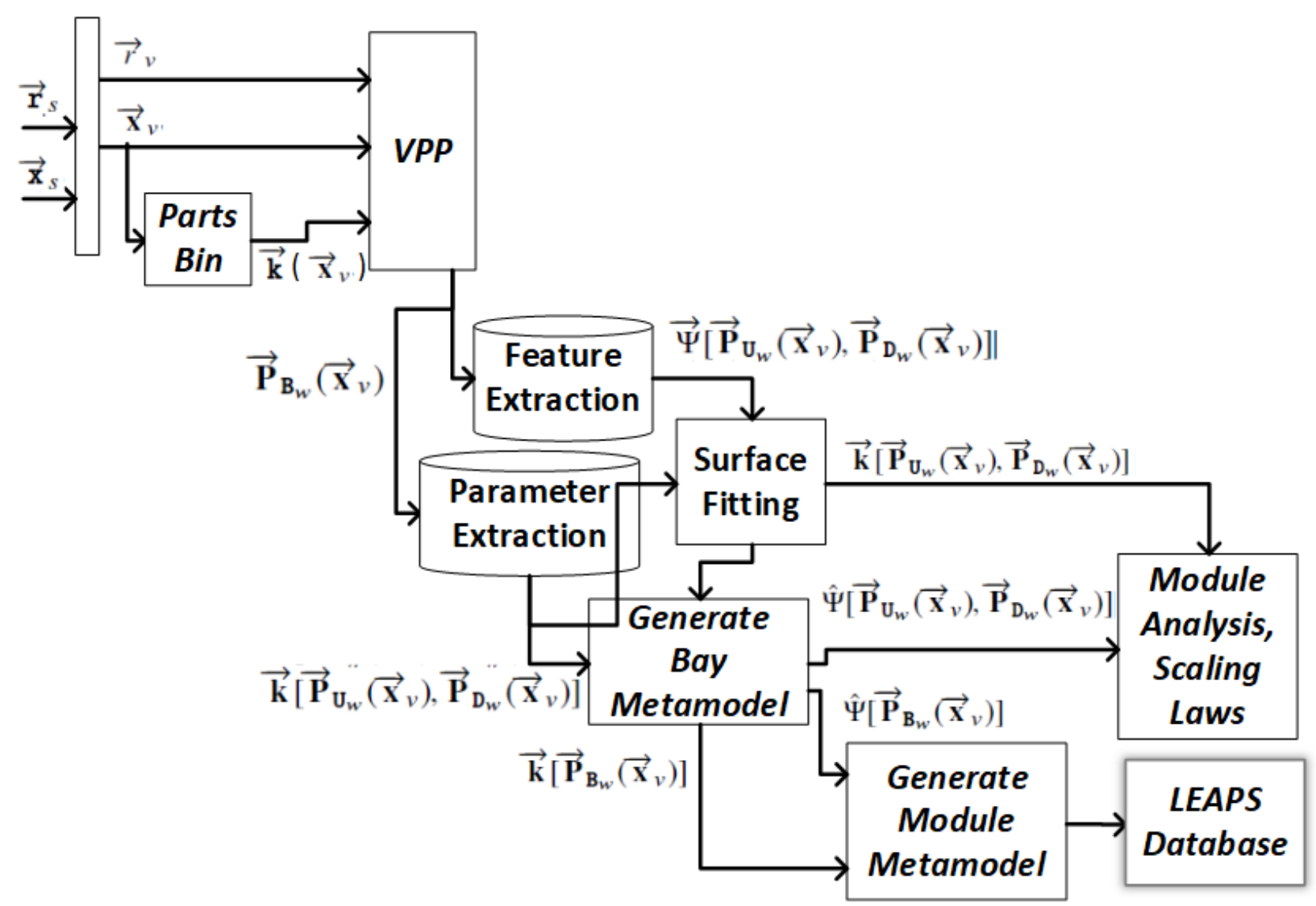

Figure 3.2: Overview of Metamodel generation process

$\overrightarrow{\mathbf{k}}\left[\overrightarrow{\mathbf{P}}_{\mathbf{U}_{w}}\left(\overrightarrow{\mathbf{x}}_{v}\right), \overrightarrow{\mathbf{P}}_{\mathbf{D}_{w}}\left(\overrightarrow{\mathbf{x}}_{v}\right)\right]$ include Drawer dimensions and weight. Examples of $\vec{\Psi}\left[\overrightarrow{\mathbf{P}}_{\mathbf{U}_{w}}\left(\overrightarrow{\mathbf{x}}_{v}\right), \overrightarrow{\mathbf{P}}_{\mathbf{D}_{w}}\left(\overrightarrow{\mathbf{x}}_{v}\right)\right]$ include LRU stresses, Drawer level losses, pressure drop $(\Delta P)$, temperature increase $(\Delta T)$, etc. The Surface Fitting function of Fig. 3.2 performs least square surface fitting of $\vec{\Psi}\left[\overrightarrow{\mathbf{P}}_{\mathbf{U}_{w}}\left(\overrightarrow{\mathbf{x}}_{v}\right), \overrightarrow{\mathbf{P}}_{\mathbf{D}_{w}}\left(\overrightarrow{\mathbf{x}}_{v}\right)\right]$ so that behavioral models for Module and Drawer, $\hat{\Psi}\left[\overrightarrow{\mathbf{P}}_{\mathbf{U}_{w}}\left(\overrightarrow{\mathbf{x}}_{v}\right), \overrightarrow{\mathbf{P}}_{\mathbf{D}_{w}}\left(\overrightarrow{\mathbf{x}}_{v}\right)\right]$ and Bay, $\hat{\Psi}\left[\overrightarrow{\mathbf{P}}_{\mathbf{B}_{w}}\left(\overrightarrow{\mathbf{x}}_{v}\right)\right]$, can be derived as a function of those subsets of $\overrightarrow{\mathbf{x}}_{v}$ that will form Module interfaces that are dynamic. The metamodel data is constructed from these inputs and a metamodel that is a function of Module design space inputs and performance vs. design space is generated by reconstructing the process by which $\overrightarrow{\mathbf{k}}\left[\overrightarrow{\mathbf{P}}_{\mathbf{B}_{w}}\left(\overrightarrow{\mathbf{x}}_{v}\right)\right]$ produced in a more efficient form from the metadata inputs. This metamodel is then programmed into the LEAPS database component.

The metamodel generation process has an additional step, which is to analyze the Pareto front performance space and functions in order to produce metrics for performance as a function of design space changes. If the Surface Fitting is expanded to include least square curve fitting to 
the parameters surfaces $\overrightarrow{\mathbf{k}}\left[\overrightarrow{\mathbf{P}}_{\mathbf{U}_{w}}\left(\overrightarrow{\mathbf{x}}_{v}\right), \overrightarrow{\mathbf{P}}_{\mathbf{D}_{w}}\left(\overrightarrow{\mathbf{x}}_{v}\right)\right]$ to the design space $\overrightarrow{\mathbf{x}}_{v}$ and then derive scaling laws that estimate the impacts of design changes on future metrics. This is the means by which this entire process will provide insights to the entire shipbuilding and associated technical community on the real impacts of technological insertions into the shipboard equipment. 


\section{CHAPTER 4}

\section{VIRTUAL PROTOTYPING PROCESS}

The main function of virtual prototyping process is to obtain an optimal system design for the cabinet base of the ship. The process goes through different possible options of arranging LRUs such as PEBBs, inductors, high frequency transformers, disconnect switches, etc. into drawers[3]. These drawers fit into a cabinet bay which serves as a basic building block upon which systems will be ultimately built in S3D.

MMC gives flexibility to stack sub modules of H-Bridges to achieve higher voltages for a MVDC System. This thesis focuses on generation of the a range of PGM designs over the design space (MVDC bus voltage, PEBB type, inlet water coolant temperature and generator frequency) according to objective functions that will produce a $\rho-\eta-\gamma$ optimized designs. Step 1 of the virtual prototyping process (VPP-Step 1) is described in the flowchart shown in Fig.4.1. According to this process, the initial $I_{s}, f_{s w}, I_{d c}, I_{a r m}$, device losses, $C_{m i n}, L_{a r m}$ and $L_{f}$ are calculated from the system model and design constraints. The resultant values for $I_{\text {arm }}$ and $L_{\text {arm }}$ become constraints for the inductor optimal design process. The design space and constraints, shown in Table 4.1 are the inputs to the inductor design process (VPP-Step2). For each design space point, multiple best options for the arm inductor designs are generated. Once the arm inductor designs are generated, the physical characteristics (size, weight and losses) are know and, together the PEBBs physical characteristics, the attributes for the LRUs are known. The LRUs are then arranged into drawers, which include system level allocations such as dielectric stand-off, thermal management, frame structure, cabling, etc., as described in [15]. Drawers are arranged into compartments and compartments are arranged into bays as shown in Fig. 1.2 and Fig. 1.3 in order to construct a single phase of the MMC. The total MMC system is constructed from bay building blocks in order to generate a range of PGM designs over the design space. This final process (VPP-Step3) is shown in Fig. 4.2. 


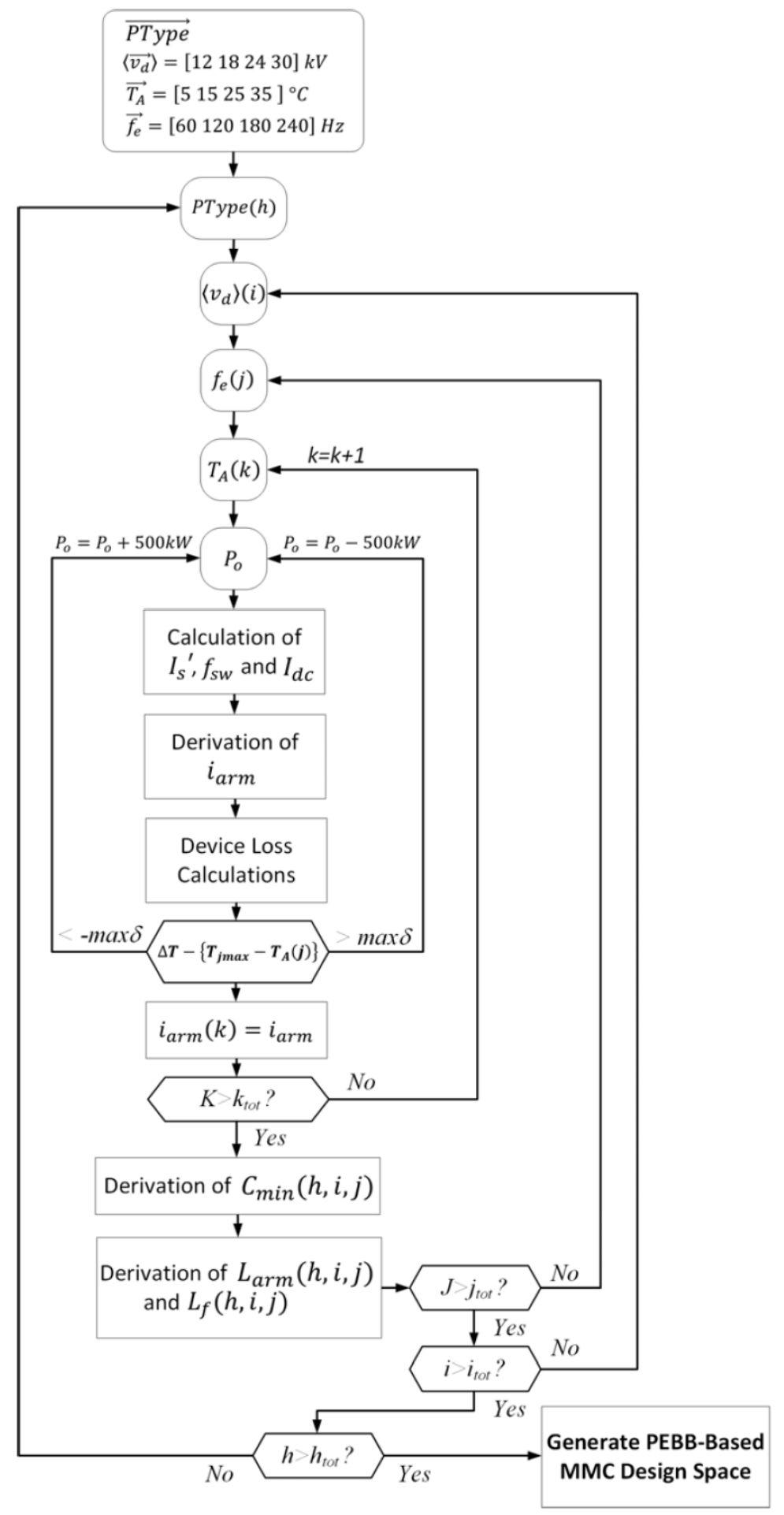

Figure 4.1: Virtual Prototyping Process(VPP-Step1) 
Table 4.1: Design Space and Constraints for Virtual Prototyping Process(VPP-Step2)

\begin{tabular}{|c|c|}
\hline \multicolumn{2}{|c|}{ Design Space } \\
\hline Design Variables & Ranges Considered \\
\hline PEBB Types & SiC MOSFET based SM-PEBB 1000, PEBB 6000 \\
DC Voltage Values (kV) & $12-30$ \\
Generator Frequency (Hz) & $60,120,240,360$ \\
Inlet Cooling Water Temperature (C) & $5-35$ \\
\hline \multicolumn{2}{|c|}{ Design Constraints } \\
\hline Design Variables & Ranges Considered \\
\hline$I_{\text {arm }}(\mathrm{A})$ & $148.89-473.41$ \\
$L_{f}(\mathrm{H})$ & $0.005-0.02$ \\
$L_{\text {arm }}(\mathrm{mH})$ & $0.32-20$ \\
\hline
\end{tabular}

The unique feature in the thesis is the arm $\left(L_{\text {arm }}\right)$ inductor optimization (explained in section 3$)$. The use of evolutionary algorithms such as the genetic algorithm (GA) results in a set that displays trade-offs among the best possible size, mass and power loss of the inductors. The process of GA being incorporated into the overall process is shown in Fig. 4.3. Moreover, this set of inductor options can be coupled as input data for the cabinet optimization algorithm, as shown in Fig. 4.2 (COA). COA follows Navy specifications for drawers, cabinets and the bay design. Ultimate results, displayed as a 3D Pareto optimal front, are shown in Fig.4.5.

\subsection{Optimization of Arm and Filter Inductors}

Furthering an understanding of inductor optimization necessitates a categorization of design metrics. The following subsections of this thesis discuss the characteristics of the core and winding that define the specification requirements of the inductor and impact the three aforementioned objectives. 


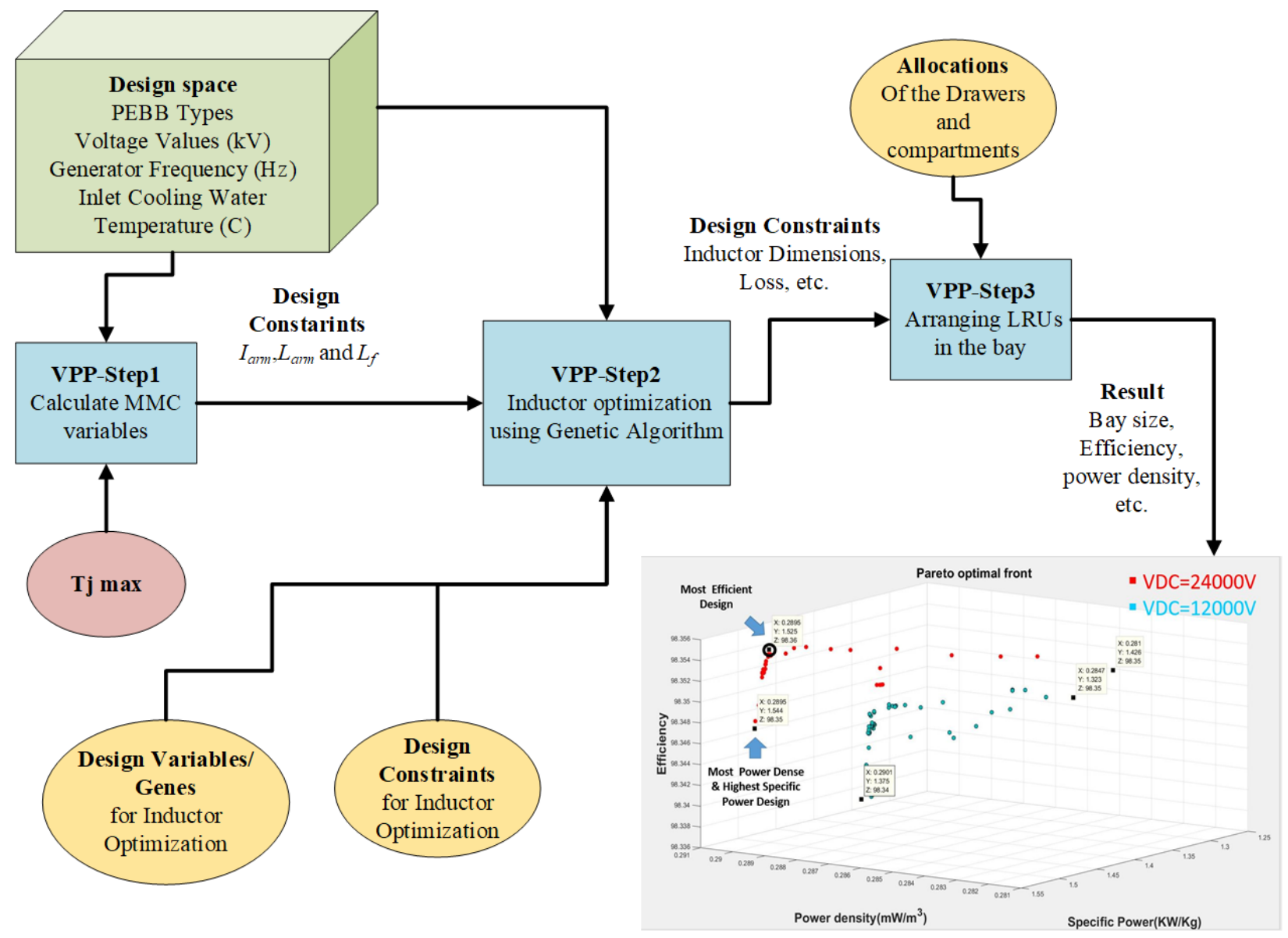

Figure 4.2: Overall Virtual Prototyping Process including VPP-Step3 


\subsection{Design Metrics of the Inductor}

\subsubsection{The Magnetic Core: Storing Energy}

An inductor's ability to reduce noise in the current waveform correlates with its potential energy capacity. And by Faraday's Law, the voltage that can be stored by the component is dependent upon the time-variant flux that stems from the magnetic field generated by the inductor.

Based upon this idea, there are two approaches to compute the electromotive force. One would be to evaluate the strength of said magnetic field; with a magnetized core, the flux density $\vec{B}$ would be a function of the initial field intensity $\vec{H}$ :

$$
\vec{B}=\mu \vec{H}
$$

where $\mu$ is further defined as the following:

$$
\mu=\mu_{0} \mu_{r}
$$

$\mu_{r}$ is the relative permeability of the core material. Unfortunately, the anhysteretic B-H characteristic for many of these magnetic materials - especially the transition between the unsaturated and saturated regions - would require extensive curve-fitting to model accurately, complicating $\mu_{r}$. To take the other approach, therefore, would involve finding the time-variant flux based on self inductance:

$$
\lambda=L i
$$

A critical assumption was made in incorporating this method into the simulation: the range of current values was restricted to be within the linear region of the $\lambda-i$ characteristic. Therefore, $L$ could be computed as the slope between two $\lambda-i$ points:

$$
L=\frac{\left.\lambda\right|_{i=i_{\max }}-\left.\lambda\right|_{i=i_{\min }}}{i_{\max }-i_{\min }}
$$


By setting self-inductance to be the benchmark for inductor energy storage and arriving at (4), the last set of formulas would involve finding flux linkage values for the maximum and the minimum current. Determining flux for a given current value can be achieved through a magnetic equivalent circuit (MEC), which helps to visualize how the field could interact the core. Specifically, MEC expressions involving MMF $(F)$, flux, permeance, $(P)$, reluctance $(R)$ is expressed by a reflection of Ohms Law reproduced from [40]:

$$
\begin{gathered}
F=\phi_{M} R \\
F=\phi_{M} R(\xi) \\
R(\xi)=\left\{l A \mu_{H}\left(\frac{F}{l}\right), \xi \sim F \frac{l}{A \mu_{B}\left(\frac{\phi_{M}}{A}\right)}, \xi \sim \phi_{M}\right.
\end{gathered}
$$

The first equation describes magnetic field lines passing through a magnetically linear material (such as air) while the second accounts for a (nonlinear) magnetic material; the reluctance is described in (7) using the evaluated length of the flux path 1, the cross-sectional area through which the flux passes A, and permeability in terms of flux density or field intensity.

Symbolic notations in an MEC correspond with those in an electric circuit; for example, the element that flows through the magnetic circuit is flux. Nodes are created at surfaces where the MMF drop is negligible;. A branch containing an element with reluctance occurs whenever the magnetic flux passes through a different material or surface area.

As the flux is implied by the equations (5) and (6) to be strongest when the reluctance is weakest, an MEC could be drawn in consideration of a set flux path or multiple flux paths in which the field lines can best permeate [40]. Such an MEC would be severely lacking in accuracy, however, since the most common flux path through the core components and the designated air gaps is not the only possible flux path. With such high current values being inputted into the inductor, the MMF source enables magnetic field lines to forge other routes in spite of the high reluctance values at certain regions of the inductor. One of these alternate paths that could be taken is called a fringing flux; the field lines traverse the $U$ and the I part of the core, but at the air gap 
the field lines take a more radial course, starting and ending at different surfaces of the core in comparison to most direct flux path.

The other set of alternate routes that could be taken is categorized under leakage flux. In this case, the field lines do not even traverse the entire core. And for some pathways the field lines only pass through part of the winding, which then must be reflected by taking a fraction of the total MMF that could be generated. Two critical equations are derived by [40] - each describing the stored energy due to the time-variant magnetic field lines that travel along a certain leakage path; one substitutes the definition of self-inductance into Faradays Law to express the energy in terms of permeance while the other combines Faraday's Law, Ampere's Law, and the definition of flux linkage to relate energy with the volume of the winding $V$ as well as the field intensity and flux density associated with said path $\left(H_{p}\right.$ and $\left.B_{p}\right)$.

$$
\begin{gathered}
E=\frac{1}{2} P N^{2} i^{2} \\
E=\frac{1}{2}{ }_{V} B_{p} H_{p} d V=\frac{1}{2} \mu_{V} H_{p}^{2} d V
\end{gathered}
$$

To avoid a direct evaluation of these expressions in a time domain, (8) and (9) are equated. From there, the $P$ (and $R$ ) are determined while taking into consideration how much of the flux path passes through the winding (which affects the value of $\mathrm{H}$ ).

The MEC that was ultimately used for the inductor optimization process was derived by [40] and involved the most direct flux path, fringing flux paths consisting of the inner corner and the three outer corners between the $\mathrm{U}$ and I part of the core, and horizontal and vertical leakage flux paths; alternate flux paths were denoted by parallel branches.

It should be briefly noted that because of availability of existing framework code for a UI core, this geometry was used for inductor optimization; future updates to the virtual prototyping method could include a comparison of various core geometries (most notably the UU-core design). For now, the process of modelling an MEC with a UI core can be found in the supplementary MATLAB codes of [40]. 


\subsubsection{The Magnetic Core: Power Loss}

In addition to energy storage, core material and geometry factor heavily in the energy dissipated by the inductor. One type of core power loss occurs when the $\mathrm{AC}$ component of the current waveform causes the core to magnetize and demagnetize continually. Energy is spent to compensate for what is known as the hysteresis effect as the external magnetic field attempts to align the magnetic dipole moments of the core [41]. To elaborate, once the core material has been magnetized, the process of demagnetizing or magnetizing the core to align with different initial field direction becomes much more difficult [41]. The anhysteretic (without hysteresis) curve can no longer serve to explain the relationship between the field intensity and the resultant flux density; instead, external B-H curves must be developed. And the area of these outer B-H curves is proportional to the power loss density of the core material due to hysteresis. For the purposes of this study, a behavioral method better known as the modified Steinmetz equation (MSE) and outlined in [40] was applied to the calculation of power losses due to hysteresis. The following formulas demonstrate how MSE was added to the inductor design process:

$$
\begin{gathered}
p_{\text {hys }}=f e_{B H} \\
e_{B H}=\frac{k_{h}}{f_{b}}\left(\frac{f_{e q}}{f_{b}}\right)^{\alpha-1}\left(\frac{\Delta \mathrm{B}}{2 B_{b}}\right)^{\beta} \\
f_{e q}=\frac{2}{(\Delta \mathrm{B})^{2} \pi^{2}} \int_{0}^{T}\left(\frac{d B}{d t}\right)^{2} d t
\end{gathered}
$$

$f$ denotes the number of times that the $\mathrm{B}-\mathrm{H}$ characteristic is traversed per second and uses the fundamental frequency of the flux density waveform, while $f_{e q}$ is the equivalent frequency of the waveform. $k_{h}, \alpha$, and $\beta$ are all constants that pertain to the core material. $f_{b}$ and $B_{b}$ match the base frequency of $1 \mathrm{~Hz}$ and the base flux density of $1 \mathrm{~T}$, respectively, and $\Delta B$ is the difference between the maximum value and the minimum value of the flux density waveform.

Consequently, the only obstacle that remains is to obtain an expression that models the flux den- 
sity waveform. What seems to be an issue is resolved, however, since a value for self-inductance was determined previously; equating the definitions of self-inductance and flux linkage by $\lambda$ yields an equation that relates $B$ in terms of the original time-variant current entering the inductor:

$$
B(t)=\frac{L}{N A_{C S}} i(t)
$$

$A_{C S}$ refers to the cross-sectional area of the base of $U$ core - through which the flux initially passes through after being generated by the winding - and $N$ is the number of turns of wire in the winding.

Alongside the effects of constant magnetization and demagnetization of the inductor, the core will develop internal currents that add to the dissipated power. Specifically, as the time-variant magnetic field lines permeate through the magnetic material, the induced voltage that contributes to the inductors storage of energy also causes current loops to form in the core around the path of the flux. In the interest of applying these current loop - better known as eddy current - losses to the inductor optimization process, an equation from [40] is reproduced here as the the following:

$$
p_{e}=k_{e} \frac{1}{T} \int_{0}^{T}\left(\frac{d B}{d t}\right)^{2} d t
$$

$k_{e}$ effectively condenses the concept of core resistivity and adding laminations to a design specification of the magnetic material. As for the flux density waveform, equation (6) that was used in hysteresis would be substituted in once again.

\subsubsection{The Magnetic Core: Materials}

With the design metrics of the core that could impact losses, volume, and mass enumerated, two material categories were considered. Ferrimagnets have one of the highest resistivities to induced currents and are demonstrated by [40] to have narrow hysteresis curves. Inductors of this core material, however, suffer from a relatively low conductivity of flux - indicated by the saturation flux density range in [40]. It is presumably for this reason that ferrites are best suited for high frequency applications in which controlling losses outweigh the ability of the field lines to permeate 
[42]. As for the silicon steel variations, which form a subset of ferromagnets, the characteristics of the material naturally offer less protection from eddy currents relative to ferrimagnets; on the flipside, silicon steel supports a relatively high saturation flux density of 1.5 to 1.8 Teslas [42][40].

It must be acknowledged that many commonly used core types - including MetGlas and nickeliron alloys, among others - were not incorporated; future developments of the inductor optimization process could expand upon the range of core materials.

\subsubsection{The Coil}

Since inductor optimization was restricted to a UI core, all designs had a single coil consisting of winding and insulation. The winding itself varied in the number of turns of wire and the crosssectional area of the conductor - specifically according to standards set by the American Wire Gauge [40]. Insulation was added to conductors in the coil through two variables: the build factor $k_{b}$ and the packing factor $k_{p f}$. The build factor (typically ranging from a value of 1.05 to 1.4) increased the cross-sectional area of a single conductor to represent the space for sheathing while the packing factor (typically varying from 0.35 to 0.65 ) indicated what percentage of the coil was occupied by the winding. The relationship between the packing and build factor was connected by the following equation reproduced from [40]:

$$
k_{p f}<\frac{\pi}{4 k_{b}^{2}}
$$

Aside from physical specifications, losses were focal in the coil analysis. To elaborate, power dissipated by the winding was conditional upon the characteristics of the current waveform. The first step in calculating losses involved extracting the DC offset of the waveform and computing losses:

$$
\begin{gathered}
P_{D C}=i^{2} r_{D C} \\
r_{D C}=\frac{V_{c l} N^{2}}{k_{p f} A_{c l}^{2} \sigma}
\end{gathered}
$$

$V_{c l}$ is the volume of the coil, $A_{c l}$ is the area of the coil, and $\sigma$ is the conductivity of the wiring. 
Skin effect due to the AC component was subsequently added to DC offset losses. Describing the phenomenon in brief, alternating current travelling through the conductor continually changes the direction of the surrounding magnetic field described by Amperes Law. This fluctuating field contributes to a time-variant flux - the same flux that is used for MEC analysis of the core; however, by Lenzs Law, the variance of flux also causes eddy current loops to form within the conductor. To obtain this form of power loss simply requires summing up the products of the resistance of the conductor due to skin effect with the corresponding RMS value of each harmonic component. Better stated in terms of mathematical expression from [40],

$$
P_{\text {skin }}=\sum_{k=1}^{n} r_{\text {skin }, k}\left(i_{R M S, k}\right)^{2}
$$

where the current waveform (and thus power loss) is approximated by an nth order Fourier series. A full explanation and derivation of $r_{s k i n, k}$ can be found in [40].

The final set of coil losses is observed when an external, time-varying magnetic field induces eddy currents in the winding; the added condition that the magnetic field not be self-induced distinguishes what is better known as the proximity effect from the previously discussed skin effect. In particular, proximity effect power losses can stem from leakage flux or fringing flux from the core as well as leakage flux from other conductors in the winding bundle.

As the various flux paths present a unique challenge for modelling proximity effect in comparison to other power dissipation calculations for the core and coil, [40] suggests classifying alternative flux paths in a manner similar to that computed for energy storage - either horizontal leakage flux, vertical leakage flux, or fringing flux and separating winding regions so that each path category dominates the region. Once the boundaries for each region have been set, all that is left is to calculate the power loss for each path. [40] derives a expression involving power loss and flux density for any specific path. And to compute this flux density, equations (8) and (9) can be equated to establish a relationship with the permeance, which is based on the geometry of the path. A more detailed explanation of the process is also provided by [40]. 


\subsection{Selection of Design Process}

The placement of the inductor in the context of the PGM-rectifier followed by an examination of the core and winding revealed unique challenges to the virtual prototyping process. To elaborate, multiple constraints would be required to bind the design space, and multiple objectives would have to be considered. Furthermore, a combination of continuous variables - gap length, length of core, depth of clearance between winding and core, among others - and discrete variables - i.e. the number of turns of wire and the type of core material - would determine inductor specifications. Such a variety and number of design factors and metrics highlighted the necessity of a versatile optimization method.

\subsubsection{Rationale behind Genetic Algorithm Selection}

The genetic algorithm (GA) is well-known for being able to handle multi-objective optimization by mimicking the processes of evolution to locate a solution (or a set of solutions); its ability to converge through probability deployed over many iterations (or generations) eliminates the need for gradients (as in Lagrange multipliers) or the dependency on a specific function for optimization (i.e. linear programming or quadratically quadratic programming)[43]. In the context of inductor design, this critical difference from other constrained optimization techniques enables discrete variables to be incorporated and analyzed just as easily as continuous variables [43].

Given the benefits of GA, it should be acknowledged according to [44] and [45] that this evolutionary algorithm lacks efficiency in comparison to particle swarm optimization (PSO), the shuffled frog-leaping algorithm (SFLA), and the krill herd algorithm (KHA); GA optimization, however, was ultimately reasoned to be more feasible in implementing inductor design given its prior applications to a wide variety of problems [43][46][47]. A more in-depth description of GA optimization found in [40] and [43] is presented by the following enumeration:

1. Initialization (first generation)

(a) A design space is formed using constants as well as a range of values presented by each 
design factor (a gene).

(b) Inductor models are created through random selection of gene values until an initial population is established.

(c) Subsequently, each member of the population is evaluated through constraints and assigned a relative fitness value according to success in maximizing or minimizing objectives.

\section{Manipulation of population}

(a) Scaling of fitness values (i.e. diversity control) is performed to prepare the population for selection as well as prevent immediate convergence of designs around a local extrema.

(b) Members of the population with high scaled fitness values are most likely to be chosen to form a mating pool; similar offspring designs with mixed gene values (crossover) are generated.

(c) Members of the population with low fitness values are most likely to be chosen to be removed (death).

(d) Part c of Initialization is repeated to form the next generation.

\section{Stopping criteria}

- If a stopping criterion is met (a max number of generations in this case), then the remaining population forms the performance space.

This performance space would contain the set of inductor configurations that have been optimized in terms of the objectives. This set is known as the pareto front, which helps to describe trade-offs between the competing objectives. Each point that is Pareto optimal signifies an allocatively efficient utilization of design variables such that an improvement in one objective function will lead to a sacrifice of another [40].

Regarding part c of initialization, (18) and (19) formulated by [40] outputs passing and failing values for less-than-or-equal-to and greater-than-or-equal-to constraints, respectively: 


$$
\begin{aligned}
& \operatorname{lte}\left(x, x_{\text {max }}\right)=\left\{1, x \leq x_{\max } \frac{1}{1+x-x_{\text {max }}}, x>x_{\text {max }}\right. \\
& \operatorname{gte}\left(x, x_{\text {min }}\right)=\left\{1, x \geq x_{\text {min }} \frac{1}{1+x_{\text {min }}-x}, x<x_{\text {min }}\right.
\end{aligned}
$$

If the sum of the constraint outputs is less than the total number of constraints - that is, if a specific configuration that does not pass all of the constraints - the inductor model automatically receives a poor fitness value.

In closing, not all mechanisms of manipulating the population were mentioned - mutation, elitism, migration, among others. What should be duly noted, however, is that many of these processes contribute to the variation of inductor models, which, in turn, supports the ability of GA to consider multiple objectives and generate an optimal set of design solutions.

\subsection{Implementation of UI-Core Inductor Design Process}

Table 4.2 provides a near-complete list of design parameters that were considered. A more in-depth explanation of each of these variables is found in [40].

Table 4.2: Design Variables for Inductor Optimization

\begin{tabular}{|l|l|}
\hline \multicolumn{2}{|c|}{ Design Space } \\
\hline Design Variables/Genes & Ranges Considered \\
\hline Core types & M19 Steel, M36 Steel, M43 Steel, M47 Steel, MN8CX, MN60LL, \\
& MN67, MN80C, 3C90 \\
Conductor type & Copper \\
Air gap (m) & $10^{-4}$ to $10^{-2}$ \\
Core length (m) & $10^{-2}$ to 0.7 \\
Width of end of U-core (m) & $2 * 10^{-3}$ to 0.7 \\
Desired conductor cross-sectional area $\left(m^{2}\right)$ & $10^{-9}$ to $10^{-3}$ \\
Desired number of turns & 1 to $10^{3}$ \\
Desired winding bundle width (\# of conductors) & 1 to $10^{3}$ \\
Desired winding bundle depth (\# of conductors) & 1 to $10^{3}$ \\
Slot clearance width from coils (m) & $10^{-6}$ to $5 * 10^{-2}$ \\
Slot clearance depth from coils (m) & $10^{-6}$ to $5 * 10^{-2}$ \\
\hline
\end{tabular}

Table 4.3 details some of the major constraints in designing the inductor. Again, [40] gives more information on each of the constraints.

Multi-objective fitness values were computed as $\frac{1}{\text { Mass }}, \frac{10^{-5}}{\text { Volume }}$, and $\frac{1}{\text { PowerLoss }}$. A scaling factor was applied to the volume to establish approximate parity with volume and power loss. Fig. 4.3 
Table 4.3: Design Constraints for Inductor Optimization

\begin{tabular}{|l|l|}
\hline \multicolumn{2}{|c|}{ Design Space Constraints } \\
\hline Design Constraints & Values \\
\hline Max allowed current density $\left(A / \mathrm{m}^{2}\right)$ & $4.96 * 10^{6}$ \\
Max height allowed $(\mathrm{m})$ & 0.5 \\
Max width allowed $(\mathrm{m})$ & 0.7 \\
Max length allowed $(\mathrm{m})$ & 0.5 \\
Max allowed mass $(\mathrm{kg})$ & $5 * 10^{3}$ \\
Max allowed power loss (W) & $6 * 10^{3}$ \\
\hline
\end{tabular}

and 4.4 highlight the organization of the genetic algorithm implementation.

Finally, Fig.4.5 displays the Pareto front of all optimal system designs found after the simulation.

\section{5 $\quad P G M-R A$ Study and Analysis}

For future ship design studies and ship system set-based design, the right selection of power converter system constraints $\overrightarrow{\mathbf{r}}_{v}$, system design space $\overrightarrow{\mathbf{x}}_{v}$ and design constants $\overrightarrow{\mathbf{k}}$ such as DC bus voltage $\left(V_{D C}\right)$, Module inlet coolant water temperature $\left(T_{A}\right)$, Module thermal management system approach ( $M_{T M S}$ and $P G M$ Generator frequency $\left(F_{e}\right)$, whill drive system level design decisions. Although the Navy has invested specifically in WBG Silicon Carbide (SiC) MOSFET FBPEBB topology implementations, in the most general sense, the Module power electronic topology $\left(M_{P E T}\right)$ informs a range of PEBB types. Variations on the PEBB can enter into the design space, such as the use of Silicon IGBT vs. SiC MOSFET devices, the voltage ratings of power semiconductor multi-chip modules, etc. Since early ship design exporations will need visibility to these decisions in order to drive research focus and, as technical readiness progress, procurement specs, it is appropriate to maintain visibility to this level of detail. Taken together, selections made within the Module level design space $\overrightarrow{\mathbf{x}}_{v}$ have an impact of system level design space $\overrightarrow{\mathbf{x}}_{s}$ considerations. Design, component selection, thermal considerations, layout analysis, dependability, reliability, sustainability, etc, have a close co-relation with the main features selection of the architectural approach vs. Module topology and vice versa. Keeping these aspects in mind, it becomes very important for the Designers to be well informed regarding the trade-offs associated with $\rho, \gamma, \eta, \sigma$ 


\section{System}

Adds to DATA constants and

constraints with a sub-structure

containing information from the

MMC Design Space (SYSTEM)

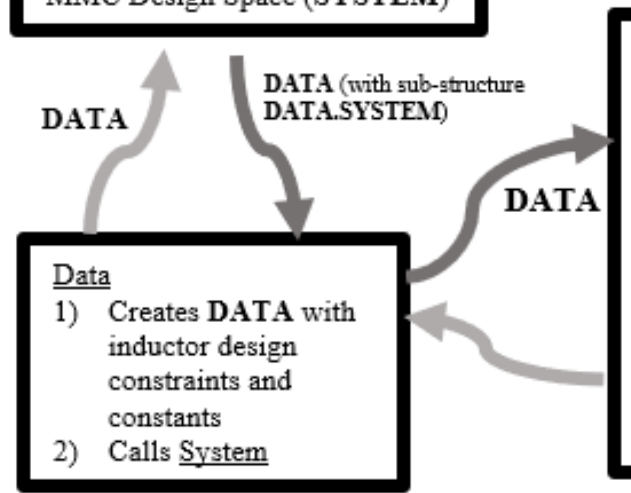

Main

1) Obtains DATA

2) Creates GA PARAMETERS that specifies how the GA will run

1) Specifies \# of generations

2) Specifies the types of operations to be performed within GA

3) Creates genes, each of which designates the range of various design specifications under consideration

3) Calls Genetic Algorithm

4) Saves results for postprocessing/display of Pareto front (not shown in diagram)

Legend:

$\longrightarrow=$ Calling program and passing parameters in

$\longrightarrow=$ Returning values

\section{Program}

\section{DATA STRUCTURE NAME}

Matrix/vectorname

DATA, Genes

\section{Fitness Function}

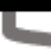

Fitness values

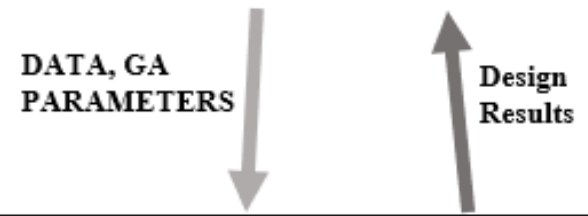

Genetic Algorithm

1) Performs an initial setup (creating the first generation of the population)

a. Obtains fitness values using fitness function

b. Scales the fitness values with random weights for consideration

2) For loop (to simulate evolving population over generations)

a. Calls a diversity control algorithm (specified by GA PARAMETERS) to prevent design models from centering on an optimum solution too quickly

b. Re-scales fitness values with diversity control under consideration

c. Calls other programs to modify population to form a new generation (i.e. mating, death, mutation)

d. Re-evaluates fitness values using fitness function Note: only the parameters and return types of the fitness function are shown.

Figure 4.3: Organization of Genetic Algorithm Implementation 

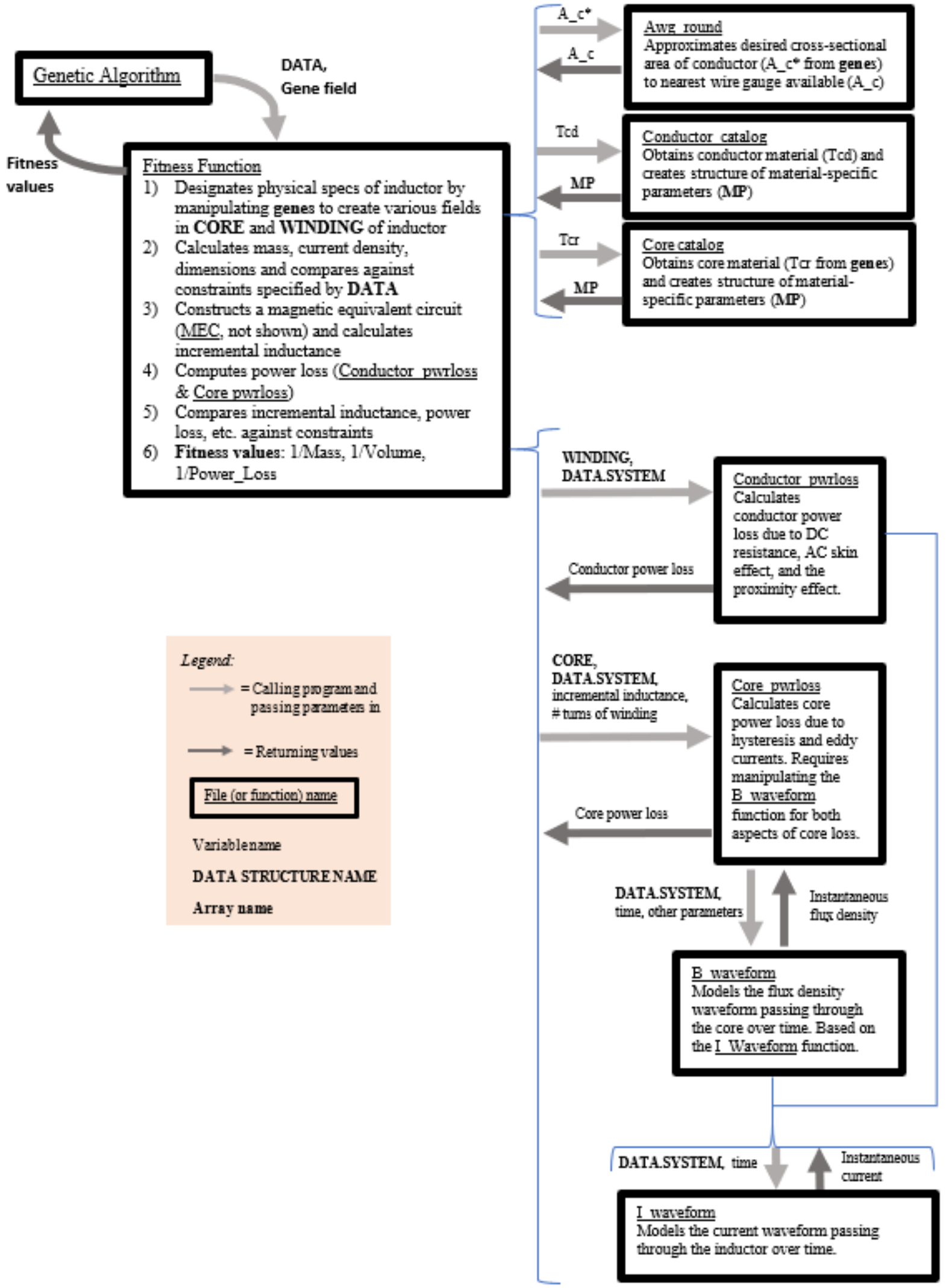

Figure 4.4: Organization of Fitness Function 


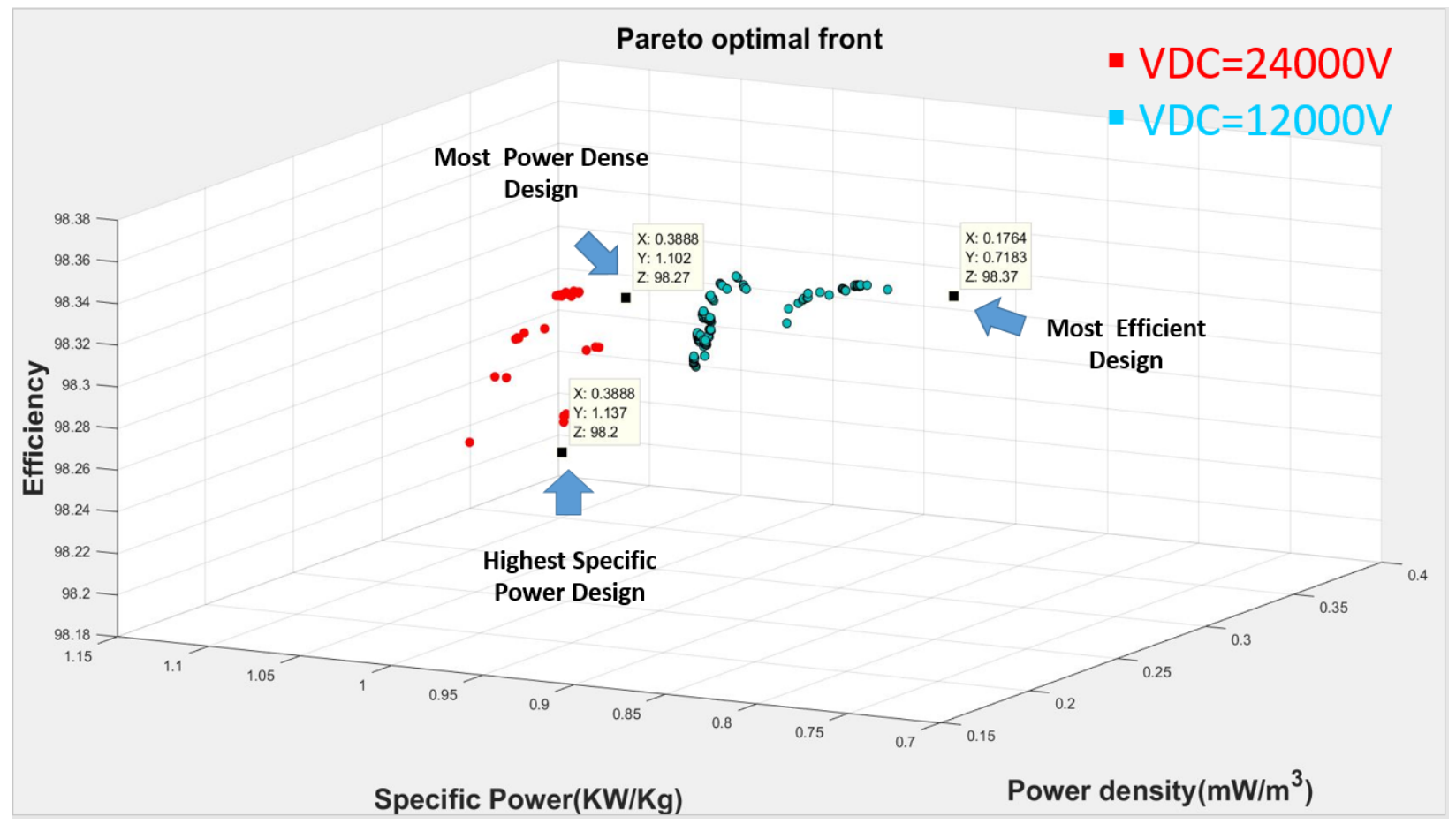

Figure 4.5: Pareto Optimal Front

and $\lambda^{-1}$. Ultimately, the naval architect will inform decision makers as to which of these objective functions is most important to a given Module.

For FB-MMC-based $P G M-R A$, Capacitor Assembly $C A_{i}$ and Inductor Assembly $I A_{i}$ optimizations play a very important role in satisfying the overall objective of system optimization. The first step of the VPP process is to derive the dependent variables associated with the MMC, calculate the losses and thermal performance from the corresponding parameters associated with the PEBB, $\vec{k}\left(P E B B_{h}\left(P E T_{j}, T M S_{k}, P D T_{l}\right)\right.$ assuming an initial intrinsic rated power, $P_{i}(0)$. The switching frequency is selected to meet a minimum level power quality, since it has little effect on the sizing of the $C A_{i}$ and $C A-i$. The impact of WBG technology to the performance will be the achievement of a higher $\vec{P}_{i}$ over the design space when compared to the Silicon IGBT and, most notably, the enabling of a $6000 \mathrm{~V}$ rated PEBB using the CREE XHV-6 10kV dual SiC module [17]. The objective function determines determines the trade-off between achieving higher power density, by operating the devices as close to the maximum junction temperature as possible, higher efficiency, which will result in operating below maximum junction temperature. The level of $P_{i}$ 
for a given design establishes derived constraints on dc link capacitance $C_{D C}$ associated with each PEBB and the required arm inductance of $I A_{i}$. The VPP allows trade comparisons between incorporating $C_{D C}$ into the PEBB assembly, $P E B B_{i}$, or into a separate $C A_{i}$. The maximum $\rho$ designs will incorporate $C_{D C}$ into the PEBB while the it is likely that the maximum $\sigma$ designs will have an external $C A_{i}$ to enable economies of scale through multi-application of $P E B B_{i}$ across a range of Modules.

The inductor and capacitor designs are optimized around the objective function $O_{(\min )}=1 / \eta$, $1 / \rho, 1 / \gamma, \lambda, 1 / \sigma$. Here, the multi-objective optimization is accomplished through an evolutionary genetic algorithm (GA). The GA enables to inclusion of a much wider design space through a gene pool, $(\vec{\Theta})$, of parameters associated with the optimal design of the inductor and its associated TMS and a range of commercially available capacitor options for the $C A_{i}$ implementation. The gene pool, $(\vec{\Theta})$, is transparent to the user of the final metamodel but final outcomes of the best designs can be exctracted after the VPP process is complete. The GA has the ability to converge through probability deployed over many iterations (or generations) and eliminates the need for gradients, which is required to design an optimal solution (size, weight and loss) of the passive components at the system level considering all the constraints. GA is very useful in avoiding getting trapped at local minima, which could lead to sub-optimal solutions. The incorporation of GA into the VPP is an elaborate design process which involves selection, mutation, cross over of genes (design space/design variables)[48]. The GA considers all the genes $(\vec{\Theta})$ and design constraints associated with Bay, Compartments, Drawers and LRUs such as the physical dimensions, aspect ratios, inductor topology, material used for the core and the winding, number of turns, etc. The result of the GA is a set of $M$ solutions that far exceed the exact number of solutions that correlate to design space $\vec{x}_{v}$. A random selection of gene values is used until to establish the initial population. Each member of the population is evaluated through constraints and assigned a relative fitness value vs. success toward minimizing objectives, diversity control, pre-selection of population with high scaled fitness values and subsequent offspring designs with mixed gene values are generated.The final stage of VPP optimally arranges the LRUs into Drawers and the Drawers into Compartments. 


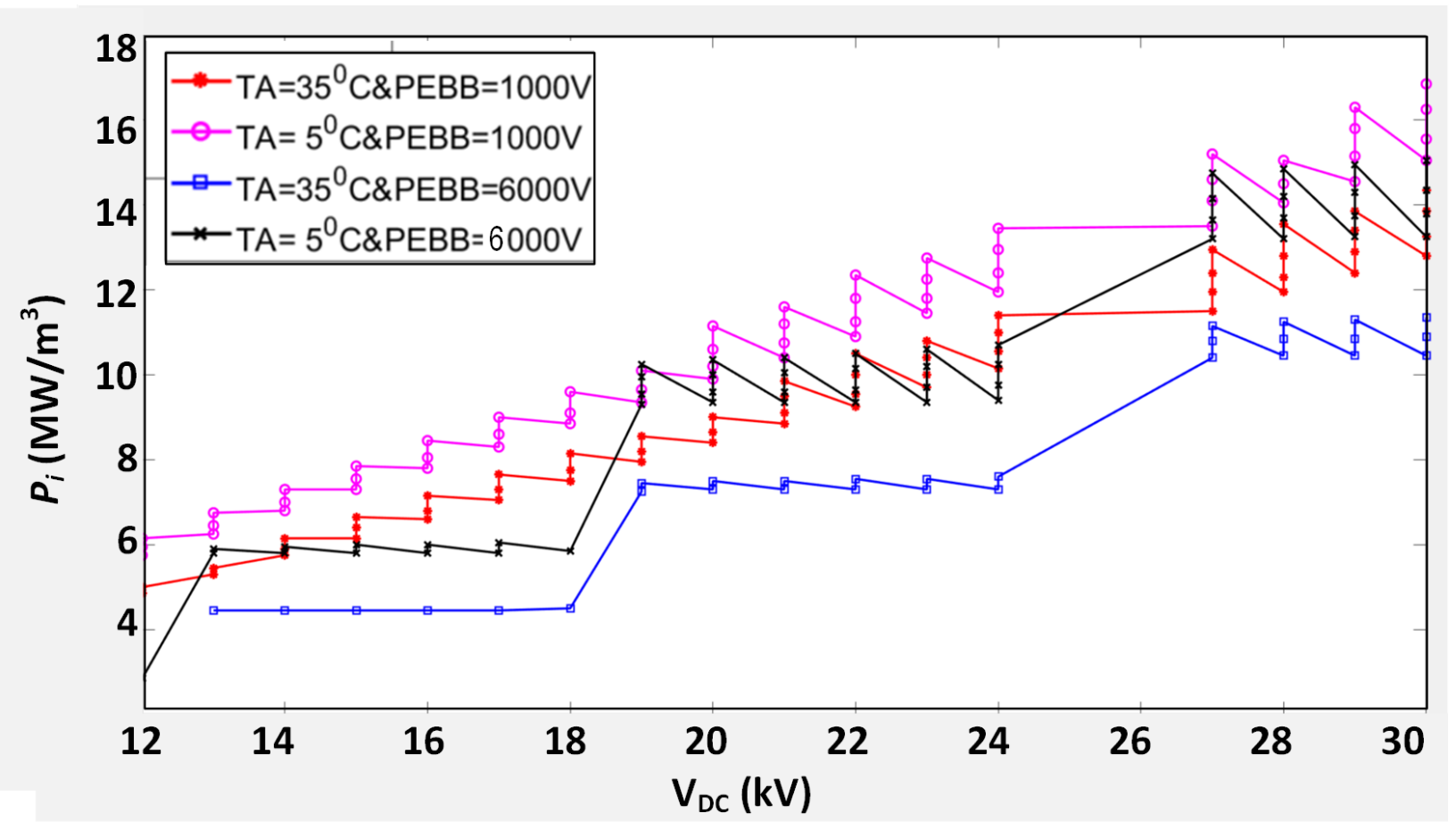

Figure 4.6: $P G M-R A$ Intrinsic Rated Power $\left(P_{i}\right)$ vs. $V_{D C}$, maximum $\rho$ designs

At this point the allocations are added for dielectric stand-off, thermal management, accessibility, structural support and cabling/interconnects.

Fig. 4.6-4.8 show the trends in $P_{i}, \rho$ and $\eta$ versus $\mathbf{v}_{D C}$ with power density maximized results for two different inlet water coolant temperature $\left(\mathbf{T}_{A}\right)=5 \mathrm{C} \& 35 \mathrm{C}$ and two PEBB types having dc link voltage ratings of $1 \mathrm{kV} \& 6 \mathrm{kV}$. The $1 \mathrm{kV}$ design utilizes CREE CAS300M17BM2 $1.7 \mathrm{kV}$ rated $\mathrm{SiC}$ MOSFET dual module. The $6 \mathrm{kV}$ design is based upon the XHV-6 dual SiC module. The two coolant temperatures represent chilled water input and non-chilled. These results show some expected trends but reveal capabilities that could only be revealed through the VPP. PGM$R A$ rated intrinsic power capabilities with an MVdc bus rating of $12 \mathrm{kV}$ are low $\left(\leq 6 \mathrm{MW} / \mathrm{m}^{3}\right)$. As expected, $P_{i}$ increases with $\mathbf{v}_{D C}$ because for each $T_{A}$ and $P E B B_{h}\left(P E T_{j}, T M S_{k}, P D T_{l}\right)$ the arm current is the same. Fig. 4.7 and 4.8 show the non-dominated optimization behavior of the VPP results. This result is to be expected due to the modular structure of the Bay and is very pronounced in the efficiency results for the 6kV PEBB. The highest efficiencies in Fig. 4.8 occur at integer increments of $6 \mathrm{kV}$. This behavior is not as pronounced in the power density results of 


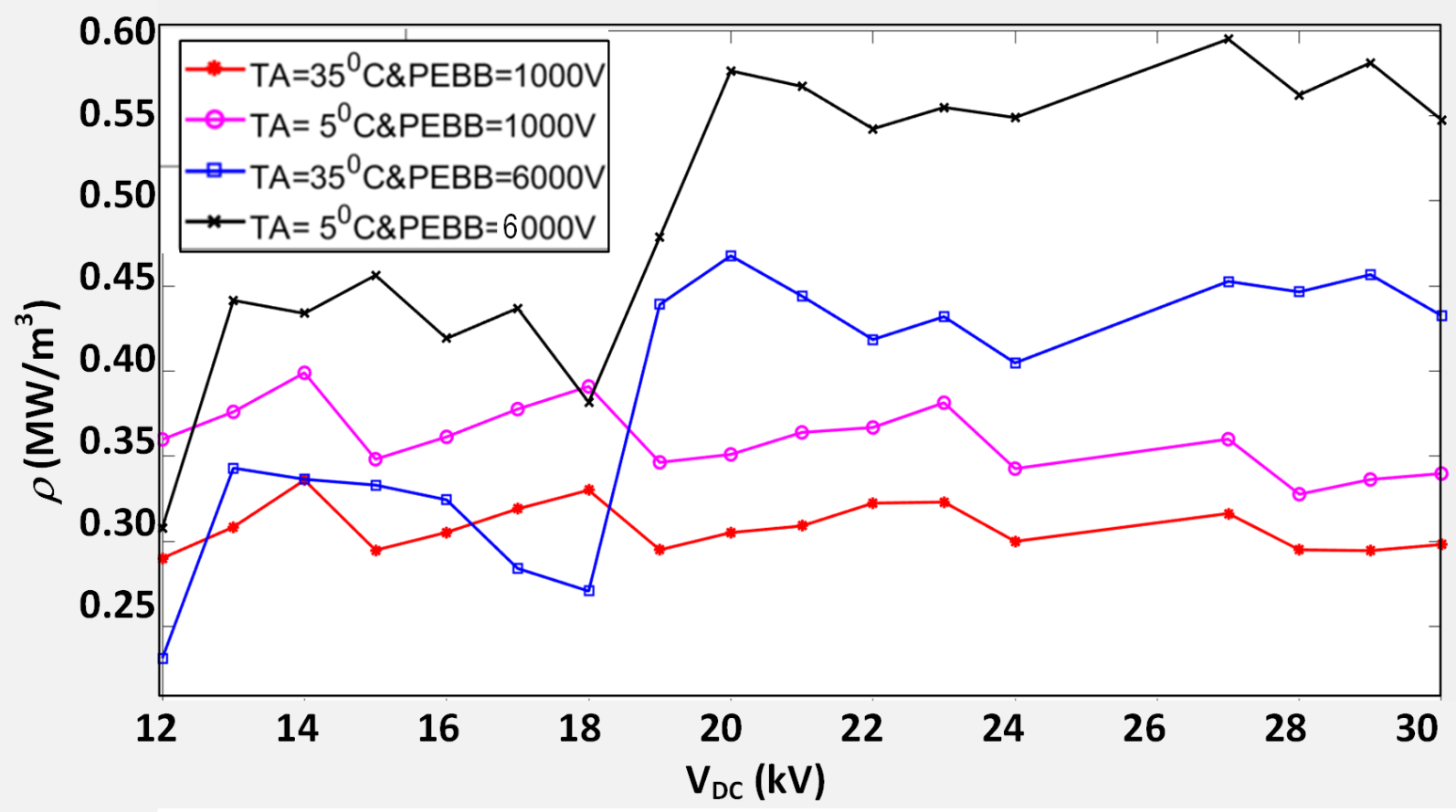

Figure 4.7: $P G M-R A$ Power Density $(\rho)$ vs. $V_{D C}$, maximum $\rho$ designs

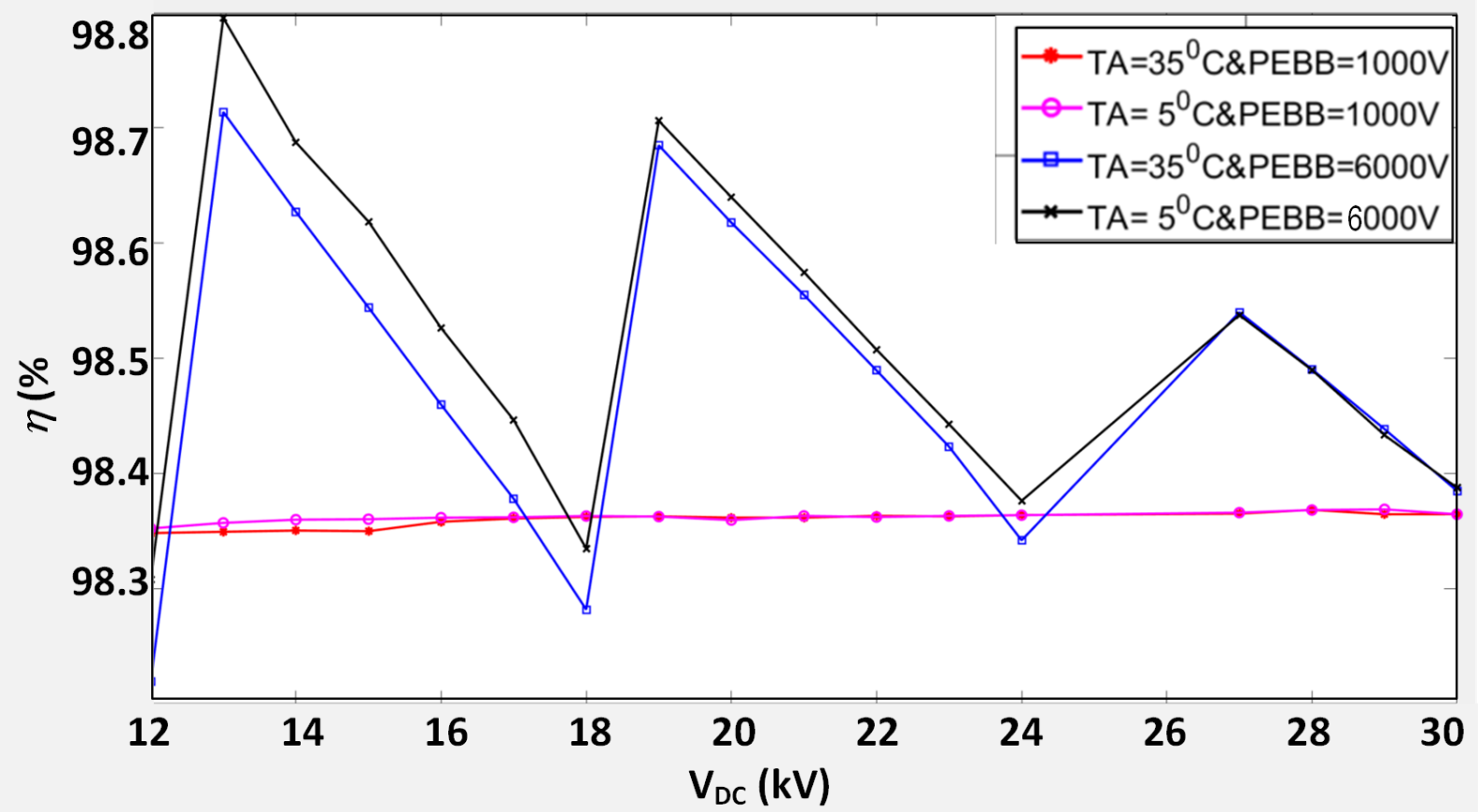

Figure 4.8: $P G M-R A$ Efficiency $(\eta)$ vs. $V_{D C}$ for, $\rho$ designs 
Fig. 4.8. This is due to impact of the arm inductor size on the overall Bay size, which dominates dominates when there are a small number of series PEBBs and as $P_{i}$ increases. The results of Fig. 4.7 are potentially significant to research direction decision makers. These results show that the $6 \mathrm{kV}$ PEBB is an undesirable approach, from a power density perspective, for $6 \mathrm{kV}$ MVdc bus voltages designs but is compelling for ship designs with MVdc bus ratings greater than $20 \mathrm{kV}$. Interestingly, the power density of the $1 \mathrm{kV}$ PEBB approach goes down as $V_{D C}$ increases. This is a strong indicator that the number of building blocks required to achieve higher voltages provides diminishing returns as the system voltage rating goes up due to the impact of the allocations at the Drawer level. 


\section{CHAPTER 5}

\section{CONCLUSION}

This thesis demonstrates the utility of the VPP for understanding the impacts of system level decisions shipboard power conversion equipment power density and efficiency through. The intended

result is that the VPP will represent to a high degree the results of an exhaustive effort to optimally design equipment for the shipboard environment. The main point of this thesis is to introduce an ontological approach capable of capturing of emerging research that will enable future shipboard designs. The process for development of a metamodel that can be programmed into a component within the LEAPS database for early ship design explorations and set-based ship system designs is also explained in detail. Future work will refine the inputs to the design space, to include ranges of topologies and thermal management methods and demonstrate the process for the other types of Modules from which IPES will be constructed. This approach is also intended to provide a means of capturing the impacts of future research into insulation systems for power converters, derivation of new rules for creepage and clearance distances and higher levels of integration between the thermal management, insulation systems, structural design and EMC hardening.

This thesis has elaborately explained the virtual prototyping process considering MMCs as framework for our research. The genetic algorithm was considered a suitable evolutionary algorithm for optimizing the inductor design. The results of the virtual prototyping process ultimately will give the most power dense, most efficient, and the best power specific design. To reiterate, Fig.4.5 demonstrates these characteristic points on the Pareto optimal fronts, where each point on the graph can give a meta-model for rapid prototyping in the ship building process. Therefore, an MMC for a MVDC type of application in a ship can have multiple initial designs for consideration of specific power, cost - $(\sigma)$, reliability - $\left(\lambda^{-1}\right)$, power density - $(\rho)$, efficiency - $(\eta)$ and weight $(\gamma)$. 


\section{REFERENCES}

[1] N. Doerry, J. Amy, and C. Krolick, "History and the status of electric ship propulsion, integrated power systems, and future trends in the us navy," Proceedings of the IEEE, vol. 103, no. 12, pp. 2243-2251, 2015.

[2] T. Ericsen, Y. Khersonsky, P. Schugart, and P. Steimer, "Pebb-power electronics building blocks, from concept to reality," 2006.

[3] R. Cuzner, M. Vygoder, and R. Siddaiah, "Power conversion and distribution equipment metamodels for dependable design of shipboard integrated power and energy systems," in 2018 IEEE International Conference on Electrical Systems for Aircraft, Railway, Ship Propulsion and Road Vehicles \& International Transportation Electrification Conference (ESARS-ITEC), IEEE, 2018, pp. 1-8.

[4] L. J. Petersen, M. Ziv, D. P. Burns, T. Q. Dinh, and P. E. Malek, "Us navy efforts towards development of future naval weapons and integration into an all electric warship (aew)," in IMarEST Engine as a Weapon (EAAW) Int. Symp. UK, 2011.

[5] J. G. Ciezki and R. W. Ashton, "Selection and stability issues associated with a navy shipboard DC zonal electric distribution system," IEEE Trans. Power Del., vol. 15, no. 2, pp. 665669, Apr. 2000.

[6] N Doerry and J Amy, "Mvdc shipboard power system considerations for electromagnetic railguns," in Proc. 6th DoD Electromagn. Railgun Workshop, 2015.

[7] R. M. Cuzner, "Power electronics packaging challenges for future warship applications," in 2015 IEEE International Workshop on Integrated Power Packaging (IWIPP), IEEE, 2015, pp. 5-8.

[8] J. D. Herbst, A. L. Gattozzi, A Ouroua, and F. M. Uriarte, "Flexible test bed for mvdc and hfac electric ship power system architectures for navy ships," in Electric Ship Technologies Symposium (ESTS), 2011 IEEE, IEEE, 2011, pp. 66-71.

[9] J. Chalfant, "Early-stage design for electric ship," Proceedings of the IEEE, vol. 103, no. 12, pp. 2252-2266, 2015.

[10] J Chalfant, M Ferrante, C Chryssostomidis, and B Langland, "Task 2: Collaborative system design environment: Integration with leaps," Technical Report submitted to the Office of Naval Research, 2013. 
[11] A. Gray, B. Cuneo, N. Vlahopoulos, and D. Singer, "The rapid ship design environmentmulti-disciplinary optimization of a us navy frigate," ASNE Day, pp. 21-22, 2013.

[12] R. Smart, J. Chalfant, J. Herbst, B. Langland, A. Card, R. Leonard, and A. Gattozzi, "Using s3d to analyze ship system alternatives for a $100 \mathrm{mw} 10,000$ ton surface combatant," in Electric Ship Technologies Symposium (ESTS), 2017 IEEE, IEEE, 2017, pp. 96-103.

[13] S. D. Sudhoff, G. M. Shane, and H. Suryanarayana, "Magnetic-equivalent-circuit-based scaling laws for low-frequency magnetic devices," IEEE Transactions on Energy Conversion, vol. 28, no. 3, pp. 746-755, 2013.

[14] R. M. Cuzner, R. Soman, M. M. Steurer, T. A. Toshon, and M. O. Faruque, "Approach to scalable model development for navy shipboard compatible modular multilevel converters," IEEE J. Emerg. Sel. Topics Power Electron, vol. 5, no. 1, pp. 28-39, 2017.

[15] R. Cuzner, S. Cruz, F. Ferrese, and R. Hosseini, "Power converter metamodeling approach for the smart ship design environment," in Electric Ship Technologies Symposium (ESTS), 2017 IEEE, IEEE, 2017, pp. 118-125.

[16] S. D. Sudhoff and R. Sahu, "Metamodeling of rotating electric machinery," IEEE Transactions on Energy Conversion, 2018.

[17] C. DiMarino, M. Johnson, B. Mouawad, J. Li, D. Boroyevich, R. Burgos, G.-Q. Lu, and M. Wang, "Design of a novel, high-density, high-speed $10 \mathrm{kv}$ sic mosfet module," in Energy Conversion Congress and Exposition (ECCE), 2017 IEEE, IEEE, 2017, pp. 4003-4010.

[18] J. Wang, Z. Shen, I. Cvetkovic, N. R. Mehrabadi, A. Marzoughi, S. Ohn, J. Yu, Y. Xu, R. Burgos, and D. Boroyevich, "Power electronics building block (pebb) design based on 1.7 kv sic mosfet modules," in Electric Ship Technologies Symposium (ESTS), 2017 IEEE, IEEE, 2017, pp. 612-619.

[19] B. Langland, R. Leonard, R. Smart, and R. A. Dougal, "Modeling and data exchange in a concurrent and collaborative design environment for electric ships," in Electric Ship Technologies Symposium (ESTS), 2015 IEEE, IEEE, 2015, pp. 388-394.

[20] K Sun, D Soto, M Steurer, and M. Faruque, "Experimental verification of limiting fault currents in mvdc systems by using modular multilevel converters," in Electric Ship Technologies Symposium (ESTS), 2015 IEEE, IEEE, 2015, pp. 27-33.

[21] R. M. Cuzner, V. Singh, M. Rashidi, and A. Nasiri, "Converter topological and solid state protective device trade-offs for future shipboard mvdc systems," in Electric Ship Technologies Symposium (ESTS), 2015 IEEE, IEEE, 2015, pp. 34-39. 
[22] M. Karami and R. M. Cuzner, "Optimal sizing of modular multi-level converters designed for shipboard applications," in Electric Ship Technologies Symposium (ESTS), 2017 IEEE, IEEE, 2017, pp. 605-611.

[23] R. Siddaiah, R. Cuzner, and T. Nguyen, "Applying a virtual prototyping process to generate pareto optimal solutions for a modular multi-level mvac to mvdc converter," in 2019 IEEE 28th International Symposium on Industrial Electronics (ISIE), IEEE, 2019, pp. 1-8.

[24] R. Cuzner and R. Siddaiah, "Derivation of power system module metamodels for early shipboard design explorations," in Electric Ship Technologies Symposium (ESTS), 2019 IEEE, IEEE, 2019.

[25] D. Kalyanmoy et al., Multi objective optimization using evolutionary algorithms. John Wiley and Sons, 2001.

[26] A. Card, M. Mazzola, and G. Henley, "Early stage design tool for marine power interconnect systems," in 2015 IEEE Electric Ship Technologies Symposium (ESTS), IEEE, 2015, pp. 349-352.

[27] J. Chalfant, B. Langland, S. Abdelwahed, C. Chryssostomidis, R. Dougal, A. Dubey, T. El Mezyani, J. Herbst, T. Kiehne, J. Ordonez, et al., "A collaborative early-stage ship design environment," CEM Publications, 2012.

[28] A. T. Jones, "Design space exploration and optimization using modern ship design tools," $\mathrm{PhD}$ thesis, Massachusetts Institute of Technology, 2014.

[29] T. Ericsen, "Future navy application of wide bandgap power semiconductor devices," Proceedings of the IEEE, vol. 90, no. 6, pp. 1077-1082, 2002.

[30] R. M. Cuzner and D. A. Esmaili, "Fault tolerant shipboard mvdc architectures," in Electrical Systems for Aircraft, Railway, Ship Propulsion and Road Vehicles (ESARS), 2015 International Conference on, IEEE, 2015, pp. 1-6.

[31] B. Langland, R. A. Leonard, H. Pais, and R. Dougal, "Automatic synthesis of detailed designs from early-stage conceptual designs," in 2017 IEEE Electric Ship Technologies Symposium (ESTS), IEEE, 2017, pp. 126-133.

[32] D. J. Singer, N. Doerry, and M. E. Buckley, "What is set-based design?" Naval Engineers Journal, vol. 121, no. 4, pp. 31-43, 2009.

[33] N Doerry and J. Amy, "Functional decomposition of a medium voltage dc integrated power system," in ASNE Shipbuilding in Support of the Global War on Terrorism Symposium, Biloxi, MS, 2008. 
[34] J Kuseian, "Naval power systems technology development roadmap-distribution a-14 may 2013,” Electric Ship Office-PM320,

[35] C. Gao, J. Jiang, X. Yang, L. Xie, and K. Cao, "A novel topology and control strategy of modular multilevel converter (mmc)," in Electrical and Control Engineering (ICECE), 2011 International Conference on, IEEE, 2011, pp. 967-971.

[36] E Kontos, R. T. Pinto, and P Bauer, "Fast dc fault recovery technique for h-bridge mmcbased hvdc networks," in Energy Conversion Congress and Exposition (ECCE), 2015 IEEE, IEEE, 2015, pp. 3351-3358.

[37] M. M. Steurer, K. Schoder, O. Faruque, D. Soto, M. Bosworth, M. Sloderbeck, F. Bogdan, J. Hauer, M. Winkelnkemper, L. Schwager, et al., "Multifunctional megawatt-scale medium voltage dc test bed based on modular multilevel converter technology," IEEE Transactions on Transportation Electrification, vol. 2, no. 4, pp. 597-606, 2016.

[38] D. Dong, Y. Pan, R. Lai, X. Wu, and K. Weeber, "Active fault-current foldback control in thyristor rectifier for dc shipboard electrical system," IEEE Journal of Emerging and Selected Topics in Power Electronics, vol. 5, no. 1, pp. 203-212, 2017.

[39] R. M. Burkart and J. W. Kolar, "Comparative evaluation of sic and si pv inverter systems based on power density and efficiency as indicators of initial cost and operating revenue," in Control and Modeling for Power Electronics (COMPEL), 2013 IEEE 14th Workshop on, IEEE, 2013, pp. 1-6.

[40] S. D. Sudhoff, Power magnetic devices: a multi-objective design approach. John Wiley \& Sons, 2014.

[41] P. A. Tipler and G. Mosca, Physics for scientists and engineers. Macmillan, 2007.

[42] C. W. T. McLyman, Transformer and inductor design handbook. CRC press, 2016.

[43] E. K. Chong and S. H. Zak, An introduction to optimization. John Wiley \& Sons, 2013, vol. 76.

[44] S. Chaturvedi, P. Pragya, and H. Verma, "Comparative analysis of particle swarm optimization, genetic algorithm and krill herd algorithm," in Computer, Communication and Control (IC4), 2015 International Conference on, IEEE, 2015, pp. 1-7.

[45] M. Yue, B. Guo, T. Hu, and X. Guo, "The research of parameters of genetic algorithm and comparison with particle swarm optimization and shuffled frog-leaping algorithm," in Power Electronics and Intelligent Transportation System (PEITS), 2009 2nd International Conference on, IEEE, vol. 1, 2009, pp. 77-80. 
[46] B. P. Loop, S. D. Sudhoff, S. H. Zak, and E. L. Zivi, "Estimating regions of asymptotic stability of power electronics systems using genetic algorithms," IEEE Transactions on Control Systems Technology, vol. 18, no. 5, pp. 1011-1022, 2010.

[47] S. Cong, G. Li, and X. Feng, "Parameters identification of nonlinear dc motor model using compound evolution algorithms," in Proceedings of the World Congress on Engineering, vol. 1, 2010.

[48] K. Deb, Multi-Objective Optimization Using Evolutionary Algorithms. John Wiley \& Sons, 2001, vol. 16. 